\title{
Nitric Oxide Induces Pathological Synapse Loss by a Protein Kinase G-, Rho Kinase-Dependent Mechanism Preceded by Myosin Light Chain Phosphorylation
}

\author{
Carmen R. Sunico, ${ }^{1}$ David González-Forero, ${ }^{1}$ Germán Domínguez, ${ }^{1}$ José Manuel García-Verdugo, ${ }^{2}$ \\ and Bernardo Moreno-López ${ }^{1}$ \\ 1'Área de Fisiología, Facultad de Medicina, Universidad de Cádiz, 11003 Cádiz, Spain, and ${ }^{2}$ Centro de Investigación Príncipe Felipe, Centro de Investigación \\ Biomédica en Red sobre Enfermedades Neurodegenerativas, Universidad de Valencia, 46012 Valencia, Spain
}

\begin{abstract}
The molecular signaling that underpins synapse loss in neuropathological conditions remains unknown. Concomitant upregulation of the neuronal nitric oxide (NO) synthase (nNOS) in neurodegenerative processes places NO at the center of attention. We found that de novo $\mathrm{nNOS}$ expression was sufficient to induce synapse loss from motoneurons at adult and neonatal stages. In brainstem slices obtained from neonatal animals, this effect required prolonged activation of the soluble guanylyl cyclase (sGC)/protein kinase G (PKG) pathway and RhoA/Rho kinase (ROCK) signaling. Synapse elimination involved paracrine/retrograde action of NO. Furthermore, before bouton detachment, NO increased synapse myosin light chain phosphorylation (p-MLC), which is known to trigger actomyosin contraction and neurite retraction. NO-induced MLC phosphorylation was dependent on cGMP/PKG-ROCK signaling. In adulthood, motor nerve injury induced NO/cGMP-dependent synaptic stripping, strongly affecting ROCK-expressing synapses, and increased the percentage of p-MLCexpressing inputs before synapse destabilization. We propose that this molecular cascade could trigger synapse loss underlying early cognitive/motor deficits in several neuropathological states.
\end{abstract}

\section{Introduction}

Synapse loss, rather than cell death, is the main factor underlying cognitive decline in Alzheimer's disease (AD) (Palop et al., 2006; Small, 2008), Parkinson's disease (PD) (Emre, 2003), Huntington's disease (HD) (Cepeda et al., 2007), multiple sclerosis (MS) (Centonze et al., 2009), and HIV dementia (Kim et al., 2008) in patients and/or in animal models. Synaptic stripping from the motoneuron surface also occurs in the progression of several motoneuron pathologies, such as amyotrophic lateral sclerosis (ALS), progressive muscular atrophy, and traumatically damaged adult motor axons (Sumner, 1975; Ikemoto et al., 1994; Sasaki and Maruyama, 1994; Ince et al., 1995; Sunico et al., 2005). Understanding the molecular basis underlying this neurodegenerative event is of central interest for the development of new therapeutic tools. Several findings point to the short half-life gas nitric oxide (NO) as a key molecule in detrimental synaptic changes. Upregulation of neuronal NO synthase (nNOS) is a common hallmark, occurring in motoneurons and reactive as-

Received Aug. 11, 2009; revised Nov. 24, 2009; accepted Dec. 1, 2009.

This work was supported by Ministerio de Ciencia e Innovación (Spain) Grant SAF2008-01415; Consejería de Innovación, Ciencia y Empresa of the Junta de Andalucía Grants PAI2005-CTS-844 and PAI2007-CTS-02606; Mutua Madrileña Foundation (B.M.-L.); and Ministerio de Ciencia e Innovación GrantSAF2008-01274 and Instituto de Salud Carlos III Grant RD06/0010/0022, Conselleria de Sanitat-Centro de Investigación Príncipe Felipe (J.M.G.-V.). We thank Dr. S. Kasparov for kindly providing initial samples of adenoviruses. We thank Elaine Lilly, PhD (Writer's First Aid), for English language revision of this manuscript.

Correspondence should be addressed to Dr. Bernardo Moreno-López, Área de Fisiología, Facultad de Medicina, Plaza Falla, 9, 11003 Cádiz, Spain. E-mail: bernardo.moreno@uca.es.

D01:10.1523/JNEUROSCI.3911-09.2010

Copyright $\odot 2010$ the authors $\quad 0270-6474 / 10 / 300973-12 \$ 15.00 / 0$ trocytes in ALS (Anneser et al., 2001; Catania et al., 2001; Sasaki et al., 2001), in neurons and/or glial cells in PD and AD (Eve et al., 1998; Lüth et al., 2000; Simic et al., 2000; Fernández-Vizarra et al., 2004), and in the striatum of HD models at early stages (Deckel et al., 2002; Pérez-Severiano et al., 2002). iNOS (inducible NOS) is also upregulated in glial cells in these diseases as well as in MS and HIV dementia (Lee et al., 2003). Additionally, NO participates in projection refinement during development (Wu et al., 1994) and in synapse loss suffered by motoneurons after motor nerve injury (Sunico et al., 2005; Moreno-López and González-Forero, 2006). However, determining whether NO synthesis is not only necessary but also sufficient to induce synapse loss remains elusive.

The small Rho GTPase RhoA and its major effector Rho kinase (ROCK) could be firm partners of NO-directed synapse elimination during neuropathological progression: (1) RhoA/ ROCK mediates neurite retraction, preventing axon growth initiation, and dendrite retraction in different neuronal types in culture (Luo, 2000, 2002); (2) activity of ROCK and protein kinase $\mathrm{G}(\mathrm{PKG})$, a downstream NO/cGMP effector, mediates semaphorin 3A-induced growth cone collapse (Dontchev and Letourneau, 2002); (3) RhoA upregulation occurs in neurons surrounding amyloid plaques and an increase in phosphorylation of RhoA/ROCK substrates is involved in $\beta$-amyloid $(\mathrm{A} \beta)$ induced inhibition of neurite outgrowth and synapse formation in a model of AD (Heredia et al., 2006; Petratos et al., 2008); and (4) RhoA/ROCK regulates fiber contraction by enhancing myosin light chain phosphorylation (p-MLC). In this way, RhoA/ROCK signaling, directly and/or indirectly activating MLC-kinase, phosphorylates MLC. It then induces actomyosin contraction and neu- 
rite outgrowth inhibition/retraction disturbing spine formation and maintenance. By decreasing synaptic connectivity during development, this mechanism has been proposed to underlie mental retardation (Newey et al., 2005).

We studied whether adenovirally directed de novo nNOS expression is "sufficient" to induce synaptic withdrawal in adult motoneurons, which normally lack this enzyme. Next, we explored in vitro the mechanism by which NO induces synaptic withdrawal and the involvement of the RhoA/ROCK pathway. Furthermore, we looked for evidence of NO involvement in synapse elimination in a model of motoneuron pathology such as motor nerve injury.

\section{Materials and Methods}

Wistar rats, obtained from an authorized supplier (Animal Supply Services, University of Cádiz, Cádiz, Spain), were cared for and handled in accordance with the guidelines of the European Union Council (86/609/ UE) and the Spanish regulations (BOE 67/8509-12; BOE 1201/2005) on the use of laboratory animals. Experimental procedures were approved by the local Animal Care and Ethics Committee.

\section{Retrograde transfection/labeling of hypoglossal motoneurons}

Replication-deficient recombinant adenoviral vectors $\left(5-8 \times 10^{11}\right.$ infective units/ml), directing the expression of enhanced green or monomeric red fluorescent proteins (eGFP and mRFP, respectively) or nNOS, were injected into the tongue to retrogradely transduce hypoglossal motoneurons (HMNs). Adenoviruses expressed eGFP, mRFP, or nNOS under the control of the human cytomegalovirus (hCMV) promoter. The Av-eGFP and Av-mRFP vectors were used as controls to test viralinduced side effects. Initial samples of adenoviruses were kindly provided by Dr. Sergey Kasparov (University of Bristol, Bristol, UK).

Adult male Wistar (250-300 g) and neonatal [postnatal day 3 (P3)] rats were anesthetized with ether diethylic. Viral solutions $(50-300 \mu \mathrm{l}$ for adults or 5-20 $\mu$ l for pups) containing $4 \%$ dimethylsulfoxide were injected by Hamilton syringe into the tip of the tongue divided into three injection points. Adult rats were allowed to survive for 5-7 d, and pups for 3-6 d.

In anesthetized animals (as above), $50 \mu \mathrm{l}$ (adults) or $20 \mu \mathrm{l}$ (pups) of the retrograde tracer aminostilbamidine methanesulfonate [FluoroGold (FG); Invitrogen], 1\% in PBS, was injected into the tongue, distributing the total volume at three injection points. Newborn and adult animals were then allowed to survive for at least 4 or $7 \mathrm{~d}$, respectively, before nerve crushing, perfusion, or extraction of acute slices.

\section{Motor nerve injury}

Adult male Wistar rats were anesthetized with chloral hydrate $(0.5 \mathrm{~g} / \mathrm{kg}$, i.p.), and the right hypoglossal (XIIth) nerve was crushed with microdissecting tweezers for $30 \mathrm{~s}$ just proximal to the nerve bifurcation, as described previously (González-Forero et al., 2004). After the lesion procedure, the nerve became translucent and it was confirmed that transection did not occur. The incision was sutured and cleaned with an aseptic solution (povidone-iodine), and the animals were allowed to survive for $7 \mathrm{~d}$ after the crushing.

\section{Extraction of brainstem slices for in vitro experiments}

Rat pups (P6-P9) were anesthetized by hypothermia (placing on ice for 10-15 $\mathrm{min}$ ) and decapitated, and their brainstems quickly removed. Dissection was in ice-cold $\left(<4^{\circ} \mathrm{C}\right)$ sucrose artificial CSF (S-aCSF) bubbled with $95 \% \mathrm{O}_{2}$ and $5 \% \mathrm{CO}_{2}$. S-aCSF composition was as follows (in $\mathrm{mm}): 26 \mathrm{NaHCO}_{3}, 10$ glucose, $3 \mathrm{KCl}, 1.25 \mathrm{NaH}_{2} \mathrm{PO}_{4}, 2 \mathrm{MgCl}_{2}$, and 218 sucrose. Transverse slices (300 to $400 \mu \mathrm{m}$ thick) were obtained using a vibroslicer (NVSL; WPI). Slices were transferred to normal oxygenated aCSF (in mм: $26 \mathrm{NaHCO}_{3}, 10$ glucose, $3 \mathrm{KCl}, 1.25 \mathrm{NaH}_{2} \mathrm{PO}_{4}, 2 \mathrm{MgCl}_{2}$, $130 \mathrm{NaCl}$, and $2 \mathrm{CaCl}_{2}$ ) and allowed to stabilize at $\sim 37^{\circ} \mathrm{C}$ for $1 \mathrm{~h}$. Next, slices were incubated for an additional period of $1-6 \mathrm{~h}\left(\sim 22^{\circ} \mathrm{C}\right)$ with aCSF supplemented with different drugs before histological processing, whole-cell patch-clamp recordings, or tissue extraction for Western blotting experiments.

\section{Histological procedures}

For confocal microscopy. Animals were anesthetized with chloral hydrate, injected intraventricularly with heparin, and perfused transcardially first with PBS, followed by $4 \%$ paraformaldehyde (PFA) in $0.1 \mathrm{~m}$ phosphate buffer (PB), $\mathrm{pH} 7.4$, at $4^{\circ} \mathrm{C}$. The brains were removed and postfixed for $2 \mathrm{~h}$ in the same fixative solution. Brainstem slices (350 $\mu \mathrm{m}$ thick) were incubated for $6 \mathrm{~h}$ with drugs, and then fixed by $30 \mathrm{~min}$ immersion in $4 \%$ PFA at $4^{\circ} \mathrm{C}$. Both brains and brainstem slices were cryoprotected by overnight immersion in $30 \%$ sucrose in $0.1 \mathrm{M} \mathrm{PB}$ at $4^{\circ} \mathrm{C}$. Serial coronal sections (30 $\mu \mathrm{m}$ thick) were obtained from both types of specimen using a cryostat and stored at $-20^{\circ} \mathrm{C}$ in a cryoprotectant solution (glycerol/ PBS, $1: 1 \mathrm{v} / \mathrm{v})$.

Immunohistochemistry was performed against synaptophysin (syn), vesicular glutamate (VGLUT2), and/or GABA (VGAT) transporters as synaptic markers; ROCK $\alpha$ and ROCK $\beta$; p-MLC; and/or the nonphosphorylated form of neurofilament $\mathrm{H}$ (SMI32) as a motoneuron marker. Sections were rinsed in PBS and immersed in $2.5 \%(\mathrm{w} / \mathrm{v})$ bovine serum albumin, $0.25 \%(\mathrm{w} / \mathrm{v})$ sodium azide, and $0.1 \%(\mathrm{v} / \mathrm{v})$ Triton X-100 in PBS for $30 \mathrm{~min}$, followed by overnight incubation at $4^{\circ} \mathrm{C}$ with different combinations of up to three antisera. Polyclonal primary antibodies used in this study were as follows: anti-syn (1:200; Zymed Laboratories), antiVGAT (1:2000; Millipore Bioscience Research Reagents), and anti-pMLC (1:125; Cell Signaling) developed in rabbit; anti-VGLUT2 (1:2500; Millipore Bioscience Research Reagents) developed in guinea pig; antip-MLC (1:250), anti-ROCK $\alpha$ (1:100), and anti-ROCK $\beta$ (1:50; Santa Cruz Biotechnology) developed in goat; and anti-SMI32 (1:8000; Covance) developed in mouse. Subsequently, the tissue was rinsed three times with PBS for $5 \mathrm{~min}$ each and incubated for $2 \mathrm{~h}$ at room temperature with the secondary antibodies, developed in donkey: anti-guinea pig, anti-goat, anti-mouse, or anti-rabbit IgGs labeled with the cyanine 2, 3, or 5 (Сy2, Сy3, Сy5) (1:400; Jackson ImmunoResearch Laboratories). Finally, sections were washed with PBS and mounted on slides with a solution containing propyl gallate $(0.1 \mathrm{~mm}$ in PBS/glycerol, 1:9 v/v). Omission of the primary antibodies resulted in no detectable staining.

Slides were analyzed using a Leica confocal microscope for fluorescence. All motoneurons were analyzed in a $z$-plane containing the nucleus. The pinhole opening ( 1 Airy unit) was the same for all experimental conditions.

For electron microscopy. Adult rats transfected with adenovirus were deeply anesthetized with chloral hydrate. The animals then received intraventricular heparin injection and transcardial perfusion with PBS, followed by 4:0.5\% PFA/glutaraldehyde (GLUT) in $0.1 \mathrm{M} \mathrm{PB,} \mathrm{pH} \mathrm{7.4,} \mathrm{at}$ $4^{\circ} \mathrm{C}$. Brainstems were removed and postfixed overnight in $4 \%$ PFA. Next, coronal sections, $30-40 \mu \mathrm{m}$ thick, were cut in a vibratome. Brainstem slices (300 $\mu \mathrm{m}$ thick), incubated for $6 \mathrm{~h}$ with drugs, were fixed by overnight immersion in 2:2.5\% PFA/GLUT in $0.1 \mathrm{M} \mathrm{PB}, \mathrm{pH} 7.4$, at $4^{\circ} \mathrm{C}$. Slices were stored in $\mathrm{PB}$ with $0.05 \%$ sodium azide at $4^{\circ} \mathrm{C}$ until electron microscopy processing.

Initially, eGFP-transfected motoneurons were identified in the slices under an epifluorescence microscope (Nikon Eclipse E800), and images were captured using a digital camera (Nikon FDX 35) for subsequent localization. Next, brainstem sections were incubated in $1 \%$ sodium borohydride in PBS for 30 min. Tissue was then rinsed in PBS and cryoprotected by immersion in $25 \%$ sucrose in PBS ( $30 \mathrm{~min}$ ), followed by six to eight quick immersions $\left(\sim 2 \mathrm{~s}\right.$ each) in 2 -methylbutane at $-60^{\circ} \mathrm{C}$. After washing, nonspecific labeling was blocked by $1 \mathrm{~h}$ incubation with $3 \%$ Aurion-BSAc (Electron Microscopy Sciences) in PBS, followed by $60 \mathrm{~h}$ incubation at $4^{\circ} \mathrm{C}$, with specific chicken anti-GFP IgY antibody (1:200; Aves Labs). Subsequently, tissue was incubated for $1 \mathrm{~h}$ at room temperature with $5 \%$ Aurion-BSAc and $0.25 \%$ coldwater fish skin gelatin in PBS, followed by $24 \mathrm{~h}$ incubation with gold-coated IgG goat antichicken antibody (1:50; Electron Microscopy Sciences). Slices were then washed with $2 \%$ sodium acetate in PBS, developed with Aurion R-Gent silver enhancement kit (Electron Microscopy Sciences), and washed again with $2 \%$ sodium acetate in PBS. Labeling was enhanced with $0.05 \%$ gold chloride solution (Electron Microscopy Sciences), stabilized with $0.3 \%$ sodium thiosulfate, and washed with PBS. Finally, slices were fixed again with $2 \%$ GLUT for $40 \mathrm{~min}$, and then stored in PB with $0.05 \%$ sodium azide at $4^{\circ} \mathrm{C}$. 
For ultrastructural studies, sections were washed in $0.1 \mathrm{M} \mathrm{PB}$ and postfixed with $2 \%$ osmium tetroxide in $0.1 \mathrm{M} \mathrm{PB}$ for $90 \mathrm{~min}$. Subsequently, the slices were rinsed with distilled water, dehydrated in graded series of ethanol including 30\% (5 min), 50\% (5 min), and 70\% (10 min), and stained for $2 \mathrm{~h} 30 \mathrm{~min}$ in $2 \%$ uranyl acetate in $70 \%$ ethanol at $4^{\circ} \mathrm{C}$. Afterward, sections were dehydrated again in graded series of ethanol including 70 (5 min), 96, and 100\%, washed with propylene oxide, embedded in Araldite resin, and left to polymerize for at least $72 \mathrm{~h}$ at $70^{\circ} \mathrm{C}$. Ultrathin sections $(70-80 \mathrm{~nm}$ thick) obtained by an ultramicrotome (Leica EM UC6) were analyzed under a FEI Tecnai Spirit electron microscope at high magnification $(43,000 \times)$ attached to a digital camera (Morada Preview). Captured images were analyzed off-line using the Image Tool software. Motoneurons were analyzed at the level of the nucleolus, except for experiments using Avs, in which analyses were performed at the level of the nucleus.

\section{Whole-cell patch-clamp recordings}

The slices were individually transferred into the recording chamber and perfused continuously (at a rate of $2 \mathrm{ml} / \mathrm{min}$ ) with different solutions at $31^{\circ} \mathrm{C}$. Whole-cell recordings were obtained from somata of HMNs visually identified based on location and characteristic size and shape (González-Forero et al., 2007) using a Nikon Eclipse CFI60 microscope equipped with infrared-differential interference contrast (IR-DIC), a $40 \times$ water-immersion objective, and an IR camera system (TILL Photonics). eGFP-transfected HMNs were identified by epifluorescence. Patch pipettes were pulled from $1.5 \mathrm{~mm}$ outer diameter borosilicate glass using a PP-830 puller (Narishige). Patch electrodes (1.5-3 M $\Omega$ resistance) contained the following (in $\mathrm{mM}$ ): $17.5 \mathrm{KCl}, 122.5 \mathrm{~K}$-gluconate, 9 $\mathrm{NaCl}, 1 \mathrm{MgCl}_{2}$, 10 HEPES, 0.2 EGTA, $3 \mathrm{Mg}$-ATP, 0.3 GTP-Tris with pH buffered to 7.2 .

Electrically evoked EPSPs were elicited with a single concentric bipolar tungsten electrode located $0.5-1 \mathrm{~mm}$ from the hypoglossal nucleus (HN) in the ventrolateral reticular formation (VLRF). The stimulus frequency was kept low enough to avoid short-term changes in synaptic responses $(0.5-1 \mathrm{~Hz})$. Stimulus duration was $50 \mu$ s. Stimulus intensity was five times the threshold stimulation. In each HMN, the stimulus threshold for EPSP was determined as the minimal stimulus intensity required to elicit a detectable EPSP in $>50 \%$ of cases. A supramaximal stimulus intensity at fivefold the threshold stimulation $(<400 \mu \mathrm{A})$ was chosen because it evoked EPSPs of maximal and fairly constant amplitude. Current-clamp recordings were obtained and low-pass Bessel filtered at $10 \mathrm{kHz}$ with a MultiClamp 700B amplifier. Data were digitized at $20 \mathrm{kHz}$ with a Digidata 1332A analog-to-digital converter and acquired using pCLAMP 9.2 software (Molecular Devices). Overall, the mean input resistance of the recorded HMNs was $76.5 \pm 6.1 \mathrm{M} \Omega$, with a range from 28.0 to $177.9 \mathrm{M} \Omega$. Only recordings with an access resistance $(<20 \mathrm{M} \Omega$ ) of $<25 \%$ of the mean input resistance $\left(R_{\mathrm{N}}\right)$ were considered acceptable for analysis. The access resistance was checked throughout the experiments, and recording was abandoned if it changed $>15 \%$. Series resistance was routinely compensated $65-75 \%$. The pipette offset potential was zeroed before the cells were patched. Leak or liquid junction potentials were not corrected.

\section{Western blotting}

Slicing and microdissection of HNs were both performed in ice-cold $\left(<4^{\circ} \mathrm{C}\right) \mathrm{S}$-aCSF supplemented with protease $(1 \mathrm{~mm}$ phenylmethylsulfonyl fluoride, $10 \mathrm{mg} / \mathrm{ml}$ leupeptin, $10 \mathrm{mg} / \mathrm{ml}$ pepstatin $\mathrm{A}, 10 \mathrm{mg} / \mathrm{ml}$ aprotinin) and phosphatase inhibitors. Tissue slices from neonatal rat pups (P7) were obtained as described above and incubated $(1,2,3,4$, or $6 \mathrm{~h})$ in carbogen-bubbled aCSF alone (control) or containing a NO donor. Microdissected HNs were homogenized in lysis buffer $[50 \mathrm{~mm}$ Tris/ $\mathrm{HCl}, \mathrm{pH}$ $7.4,1 \%(\mathrm{v} / \mathrm{v})$ Triton X-100, 0.5\% (w/v) sodium deoxycholate] supplemented with protease and phosphatase inhibitors using a $1 \mathrm{ml}$ syringe. Equal amounts of protein were processed for SDS-PAGE and immunoblotting, using a specific antibody against p-MLC and MLC developed in goat and rabbit, respectively (1:200; Santa Cruz Biotechnology). Membranes were also probed with anti- $\alpha_{1}$-tubulin antibody $(1: 250,000$; Sigma-Aldrich) as control for the total amount of protein contained in each well. Analysis was performed using the ImageJ 1.36b software from the National Institutes of Health.

\section{Drugs and treatments}

Either alone or in different combinations, the following drugs were added to the incubation bath: the long half-life NO donor (Z)-1-[2-(2-aminoethyl)$\mathrm{N}$-(2-ammonioethyl)amino]-diazen-1-ium-1,2-diolate-NO (DETA/NO) (1 mM; Sigma-Aldrich); the NO scavenger 2-(4-carboxyphenyl)-4,4,5,5tetramethylimidazoline-1-oxyl-3-oxide (carboxy-PTIO) (300 $\mu \mathrm{m}$; Tocris Bioscience); the broad-spectrum NOS inhibitor $N^{\omega}$-nitro-L-arginine methyl ester (L-NAME) (2 mM; Sigma-Aldrich); the specific soluble guanylyl cyclase (sGC) inhibitor $1 H$-[1,2,4] oxadiazolo[4,3-a]quinoxalin-1-one (ODQ) $(20$ $\mu \mathrm{M}$; Sigma-Aldrich); the cGMP permeable analog 8-Br-cGMP (0.1 mM; Sigma-Aldrich); the specific protein kinase $\mathrm{G}$ inhibitor (PKG-i) guanosine, 3',5'-cyclic monophosphorothioate, 8-4-chlorophenylthio-, Rp-isomer (Rp-8-pCPT-cGMPS) (10 $\mu \mathrm{M}$; Calbiochem); the specific inhibitor of Rho exoenzyme C3 transferase (Exo C3) $(2.5 \mu \mathrm{g} / \mathrm{ml}$; Cytoskeleton); and the specific inhibitors of ROCK trans-4-[(1R)-1-aminoethyl]-N-4pyridinylcyclohexanecar boxamide dihydrochloride (Y27632) and (S)(+)-2-methyl-1-[(4-methyl-5-isoquinolinyl)sulfonyl]-hexahydro-1 $\mathrm{H}$ 1,4-diazepine dihydrochloride (H1152) (10 $\mu \mathrm{M}$; Tocris Bioscience).

Administration of chemicals in vivo began the day of crushing or adenoviral injection into the tongue. Rats were injected intraperitoneally (adults) or subcutaneously (pups) with L-NAME $\left(90 \mathrm{mg} \cdot \mathrm{kg}^{-1} \cdot \mathrm{d}^{-1}\right)$, the inactive stereoisomer D-NAME $\left(90 \mathrm{mg} \cdot \mathrm{kg}^{-1} \cdot \mathrm{d}^{-1}\right.$; Sigma-Aldrich), the relatively specific nNOS inhibitor 7-nitroindazole (7-NI) $(30 \mathrm{mg} \cdot$ $\left.\mathrm{kg}^{-1} \cdot \mathrm{d}^{-1}\right)$, or ODQ $\left(2 \mathrm{mg} \cdot \mathrm{kg}^{-1} \cdot \mathrm{d}^{-1}\right)$. In our hands, the injected dose of L-NAME produced a transient increase in the arterial blood pressure that returned to control values $6 \mathrm{~h}$ after injection, and it was not altered by the nNOS inhibitor 7-NI. The amounts of nNOS and endothelial isoform of NOS remained as in control animals after chronic treatment with L-NAME (Moreno-López et al., 2004). Both perfusion and slice extraction were performed at least $18 \mathrm{~h}$ after the last injection of chemicals, given that constitutive NOS inhibition persisted in $>50 \%$ (MorenoLópez et al., 2004) and to fully avoid acute effects of NOS inhibition on arterial blood pressure.

\section{Statistics}

Data are expressed as the mean \pm SEM. Unpaired two-tailed Student's $t$ test or one-way ANOVA was used to compare two or more groups, respectively. When $n \leq 5$, as in electron microscopy of eGFP-transfected HMNs and immunoblot studies, the nonparametric Mann-Whitney $U$ test was applied. Statistical significance was placed at $p<0.05$. The number of analyzed HMNs and number of animals per experimental condition are indicated in figure legends.

\section{Results \\ nNOS upregulation in adult motoneurons is "sufficient" to induce excitatory, but not inhibitory, synapse withdrawal}

To investigate whether NO signaling is "sufficient" to trigger synapse loss, we used adenoviral vectors to transduce adult motoneurons with eGFP, mRFP, and/or nNOS. Injection of a mixture of two adenoviruses (Av-eGFP/Av-mRFP) into the tip of the tongue retrogradely transduced $10-50 \mathrm{HMNs}$ per animal (Fig. $1 A, B)$. Interestingly, a high frequency $(92.7 \pm 5.2 \% ; n=3$ rats) of eGFP-transduced motoneurons also coexpressed mRFP. Thus, it was highly plausible that an eGFP-positive HMN was also nNOS-cotransduced after Av-eGFP/Av-nNOS coadministration. At the times tested, inspection of coronal brainstem sections did not reveal eGFP- or mRFP-positive neurons in projection areas to the HN, such as the VLRF or in the neighboring nucleus of tractus solitarii. This rules out the possibility of a direct premotor action of the adenoviral products. One week after adenoviral injection, the linear density of syn-immunoreactive (syn-ir) puncta of eGFP/mRFP-cotransduced motoneurons (17.6 \pm 0.65 puncta $/ 100 \mu \mathrm{m}$ of membrane perimeter; $n=67 \mathrm{HMNs}$ from 3 rats) was similar to that measured in FG-backlabeled HMNs $(18.9 \pm 0.72$ puncta/100 $\mu \mathrm{m})$. This result dismisses the possibility of unwanted side effects of the transduction process on the synaptic coverage of infected HMNs. However, cotransduction 

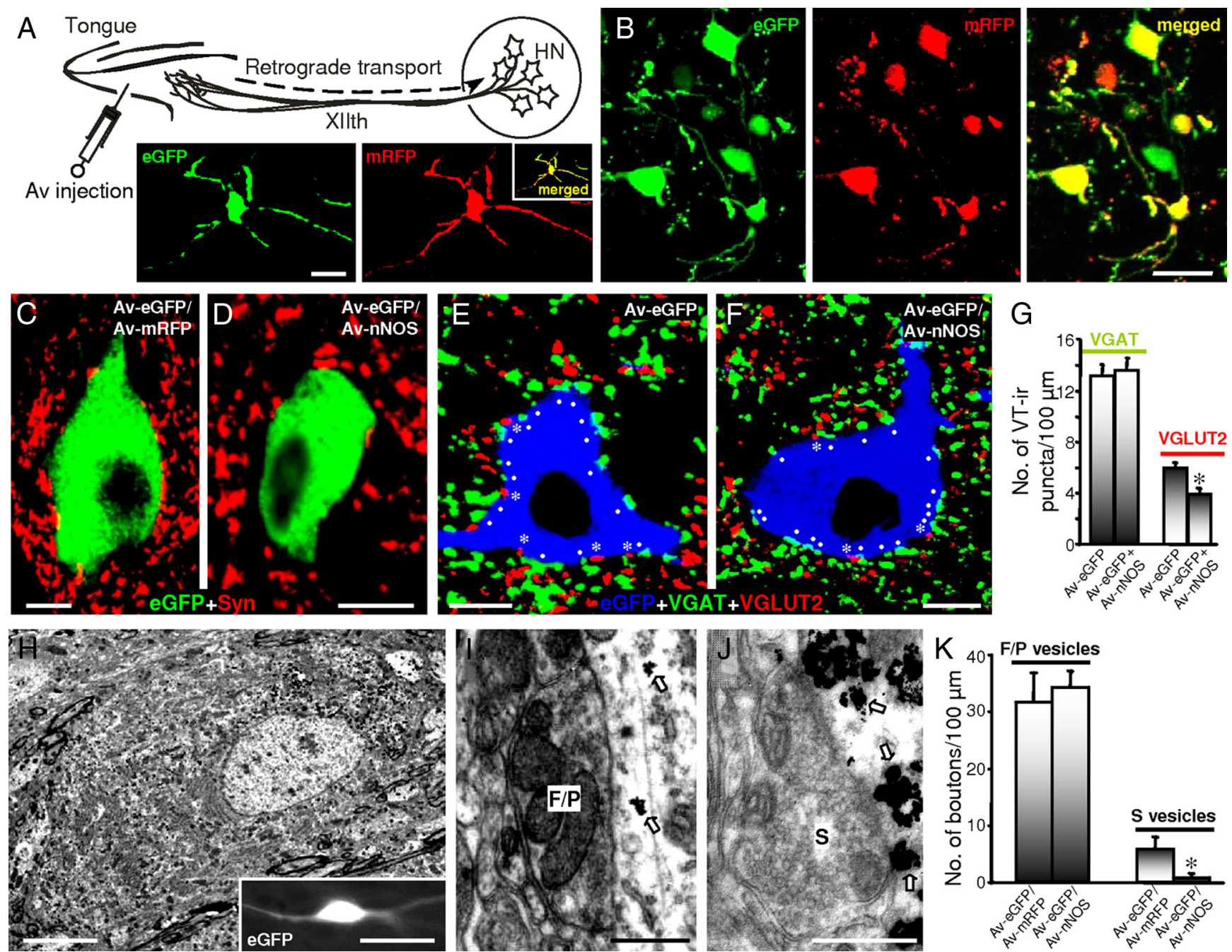

Figure 1. nNOS expression in adult motoneurons is sufficient to induce synapse loss. $A$, Top, Av injection into the tip of the tongue retrogradely transfected HMNs. Bottom, An illustrative example of retrogradely cotransduced HMN. $\boldsymbol{B}$, One week after injection of Av-eGFP/Av-mRFP, a high degree of cotransfection was observed. $\boldsymbol{C}-\boldsymbol{F}$, Syn- $(\boldsymbol{C}, \boldsymbol{D})$ or VGAT- $(\bullet)$ and VGLUT2-ir $(*)(\boldsymbol{E}, \boldsymbol{F})$ puncta around eGFP-expressing HMNs $7 \mathrm{~d}$ after injection of the indicated Avs. Secondary antibodies were labeled with Cy5 and Cy3 for immunolabeling of syn/VGAT and VGLUT2, respectively. G, Average number of the indicated vesicular transporters-ir (VT-ir) puncta per $100 \mu \mathrm{m}$ of eGFP-identified HMN perimeter at the indicated conditions (Av-eGFP, $n=38 \mathrm{HMNs}$ from 4 rats; Av-eGFP/Av-nNOS, $n=44$ HMNs from 5 rats). ${ }^{*} p<0.005$, unpaired two-tailed Student's $t$ test. $H$, A transfected HMN processed by immunogold against eGFP. This motoneuron was first selected by epifluorescence (inset). $\boldsymbol{I}, \boldsymbol{J}$, Synaptic boutons attached to the plasma membrane of a HMN identified by deposits of gold (arrows), containing $F / P(I)$ or $S(\boldsymbol{J})$ vesicles. $\boldsymbol{K}$, Average bouton frequency, characterized by the type of vesicles, attached to transfected motoneurons (Av-eGFP/Av-mRFP, $n=4 \mathrm{HMNs}$ from 4 rats; Av-eGFP/Av-nNOS, $n=3 \mathrm{HMNs}$ from 3 rats). ${ }^{*} p<0.05$, nonparametric Mann-Whitney $U$ test. Error bars indicate SEM. Scale bars: $\boldsymbol{A}, \boldsymbol{H}$, inset, $50 \mu \mathrm{m} ; \boldsymbol{B}, 100 \mu \mathrm{m} ; \boldsymbol{C}, \boldsymbol{D}, \boldsymbol{E}, \boldsymbol{F}, 10 \mu \mathrm{m} ; \boldsymbol{H}, 5 \mu \mathrm{m} ; \boldsymbol{I}, \boldsymbol{J}, 0.5 \mu \mathrm{m}$.

of HMNs with Av-eGFP/Av-nNOS induced a significant $(p<$ 0.0001, unpaired two-tailed Student's $t$ test) reduction in their synaptic coverage $(13.3 \pm 0.47$ puncta $/ 100 \mu \mathrm{m} ; n=108 \mathrm{HMNs}$ from 4 rats) (Fig. $1 C, D$ ). Results were not explained by changes in motoneuron size $(\mathrm{Av}$-eGFP/Av-mRFP, $71.0 \pm 2.56 \mu \mathrm{m}$; AveGFP/Av-nNOS, $72.0 \pm 1.39 \mu$ m perimeter).

Axonal damage of adult motoneurons is followed by vesiclecontaining spherical (S-type), but not flat/pleomorphic (F/Ptype), synapse elimination (Sumner, 1975). Given that S-type and F/P-type boutons are presumably excitatory and inhibitory, respectively (Gray, 1959; Bodian, 1966; Uchizono, 1965), we determined whether this selective action is also induced by $\mathrm{NO}$ signaling. Next, immunohistochemistry was performed against VGLUT2, to identify a subtype of excitatory synaptic inputs, and VGAT, to label inhibitory terminals. Muscle administration of Av-eGFP had no effects on the linear density of excitatory (5.98 \pm 0.40 VGLUT2-ir puncta/100 $\mu \mathrm{m})$ and inhibitory $(13.24 \pm 0.86$ VGAT-ir puncta/100 $\mu \mathrm{m}$ ) puncta apposed to transfected
HMNs relative to FG-identified motoneurons. Nevertheless, injection of Av-eGFP/Av-nNOS induced a drastic decrease in VGLUT2-ir $(-34.6 \pm 7.9 \%)$ but not in VGAT-ir puncta $(3.0 \pm 7.4 \%)$ (Fig. $1 E-G$ ).

These alterations could be the consequence of a NO-mediated disorganization of the synaptic machinery rather than the actual withdrawal of boutons. To solve this question, we studied, under electron microscopy, the synaptic coverage of transduced motoneurons identified by immunogold against eGFP (Fig. $1 H$ ). In our preparations, synaptic contacts did not show presynaptic and/or postsynaptic densities as thick as those described originally in the cerebral cortex (Gray, 1959); therefore, classification was done by type of synaptic vesicle. We distinguished between F/P-type (Fig. 1I) and S-type (Fig. $1 J$ ) boutons. In eGFP-positive motoneurons, most boutons contained clear F/P vesicles (31.8 \pm 5.08 boutons $/ 100 \mu \mathrm{m}$ ). The frequency of this type was not significantly altered by coadministration of Av-eGFP/Av-nNOS $(34.3 \pm 2.80$ boutons $/ 100 \mu \mathrm{m})$. As expected, the frequency of 

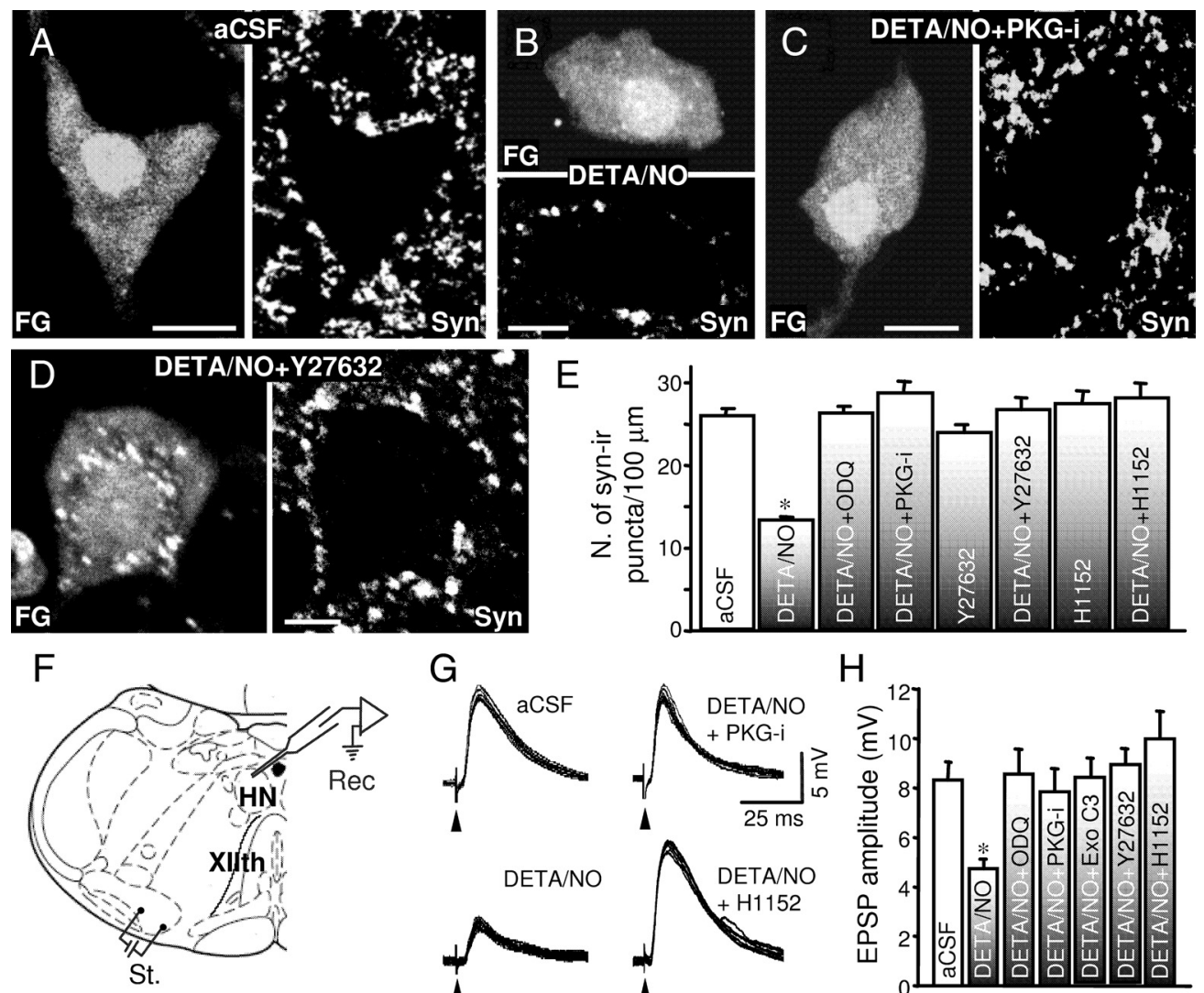

G

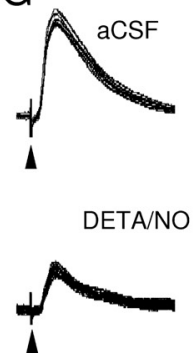

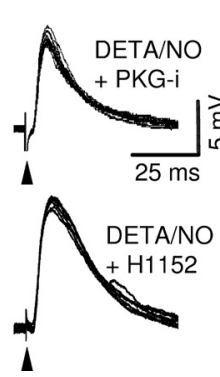

$\mathrm{H}$

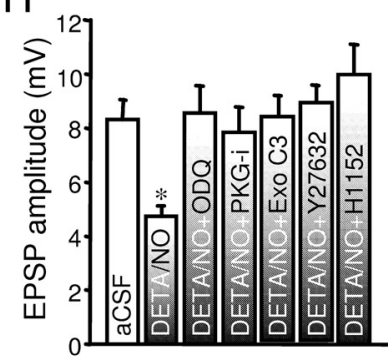

Figure 2. The NO/cGMP/PKG pathway reduces syn-ir puncta and the evoked EPSP in neonatal motoneurons in a RhoA/ROCK-dependent way. A-D, Syn-ir puncta around FG-identified HMNs obtained from slices incubated for $6 \mathrm{~h}$ as indicated. Scale bars, $10 \mu \mathrm{m}$. Secondary antibody was labeled with Cy 5 for immunolabeling of syn. $\boldsymbol{E}$, Average number of Syn-ir puncta per $100 \mu \mathrm{m}$ of FG-identified HMN perimeter at the indicated conditions (aCSF, $n=99 \mathrm{HMNs}$ from 7 pups; DETA/N0, $n=100 \mathrm{HMNs}$ from 7 pups; DETA/NO + 0DQ, $n=100 \mathrm{HMNs}$ from 7 pups; DETA/NO + PKG-i, $n=50$ HMNs from 7 pups; Y27632, $n=50$ HMNs from 4 pups; DETA/NO + Y27632, $n=50$ HMNs from 6 pups; H1152, $n=30$ HMNs from 3 pups; DETA/NO + H1152, $n=30$ HMNs from 3 pups). ${ }^{*} p<0.0001$, one-way ANOVA test relative to the aCSF condition. $\boldsymbol{F}$, Recordings (Rec) of EPSPs evoked in HMNs by electrical stimulation (St.) of the VLRF were performed by whole-cell patch clamp. $G$, Representative EPSPs (10 traces overlapped) recorded in HMNs after the indicated treatments. Membrane potential was held at $-64 \mathrm{mV}$ in all recorded motoneurons. The arrowheads point to stimulus artifact. $\boldsymbol{H}$, Average EPSP amplitude recorded in HMNs under the indicated treatments (aCSF, $n=12 \mathrm{HMNs}$ from 5 pups; DETA/NO, $n=19 \mathrm{HMNs}$ from 4 pups; $\mathrm{DETA} / \mathrm{NO}+0 \mathrm{DQ}, n=15 \mathrm{HMN}$ from 4 pups; DETA/NO + PKG-i, $n=20$ HMNs from 7 pups; DETA/NO + Exo C3, $n=14$ HMNs from 5 pups; DETA/NO + Y $27632, n=20$ HMNs from 6 pups; DETA/NO + H1152, $n=14$ HMNs from 4 pups). ${ }^{*} p<0.0001$, one-way ANOVA test relative to the aCSF condition. Error bars indicate SEM.

S-type boutons was selectively reduced $(-85.4 \pm 6.51 \%)$ after transduction with Av-eGFP/Av-nNOS (0.9 \pm 0.86 vs $5.9 \pm 2.17$ boutons $/ 100 \mu \mathrm{m}$ in controls) (Fig. $1 \mathrm{~K}$ ). These data strongly support that nNOS upregulation in adult motoneurons is sufficient to induce the detachment of excitatory, but not inhibitory, synapses. Strikingly, ultrastructural analysis revealed that synaptic frequency of S-type boutons was reduced to a greater extent than VGLUT2-ir puncta detected by immunofluorescence. The reasons for this apparent discrepancy may lie in the fact that: (1) under confocal microscopy, we only analyzed puncta for VGLUT2, but not for VGLUT1; (2) single VGLUT2-ir puncta does not necessarily represent individual synaptic boutons; and (3) it is likely that our quantitative analysis of VGLUT2 staining on confocal images underestimates synaptic stripping because it does not allow to discriminate between boutons contacting the neuronal membrane and those that have been detached but remain in proximity to the soma.

Long-term action of NO/cGMP/PKG pathway reduces syn-ir puncta and evoked EPSPs in neonatal motoneurons

To characterize molecular partners acting downstream from NO in synaptic stripping, we incubated brainstem sections obtained from rat pups (P6-P9) with several regulators of the NO/cGMP/ PKG cascade. The characteristic uninterrupted rhythmic dis- charge of adult HMNs in vivo would be expected to support a sustained production of NO in nNOS-transduced motoneurons. In an attempt to mimic this microenvironment, slices containing FG-labeled HMNs were preincubated $(\sim 6 \mathrm{~h})$ with a long half-life NONOate, DETA/NO, before immersion in the fixative solution. The linear density of syn-ir puncta apposed to DETA/NOsuperfused motoneurons was reduced $(-48.9 \pm 1.7 \%)$ relative to control HMNs $(25.9 \pm 0.93$ puncta $/ 100 \mu \mathrm{m} ; \sim 6 \mathrm{~h}$ incubation in aCSF) (Fig. $2 A, B, E$ ). As in adults, results could not account for changes in the size of analyzed motoneurons (control, $61.9 \pm$ $1.49 \mu \mathrm{m}$; DETA/NO, $63.1 \pm 1.25 \mu \mathrm{m}$ in perimeter). Reduction in the frequency of syn-ir puncta was avoided in slices coincubated with DETA/NO plus either the sGC inhibitor ODQ or the PKG-i Rp-8-pCPT-cGMPS (Fig. 2C,E). Thus, NO-induced reduction in synaptic puncta apposed to motoneurons was mediated via sGC and PKG activation.

Functional correlations were studied by analyzing whole-cell patch-clamp recordings of the EPSPs evoked in HMNs by electrical stimulation of the VLRF (Fig. 2F). Corroborating histological findings, DETA/NO (for 5-6 h) induced a decrease $(-43.1 \pm$ $4.5 \%$ ) in the amplitude of the orthodromic excitatory synaptic potential relative to the aCSF incubation alone (Fig. $2 G, H$ ). Although the rise time of EPSPs (10-90\% of peak amplitude) tended to be slightly longer in DETA/NO-treated HMNs (con- 
A

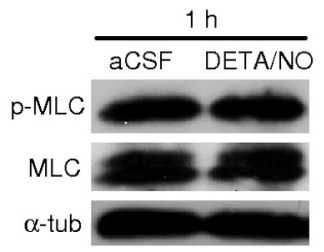

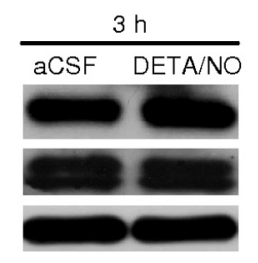

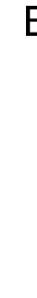

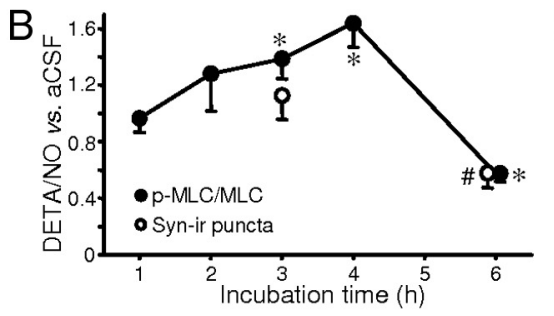

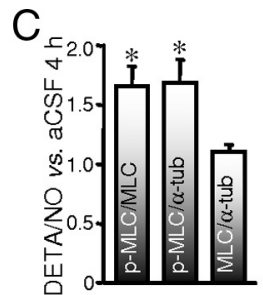

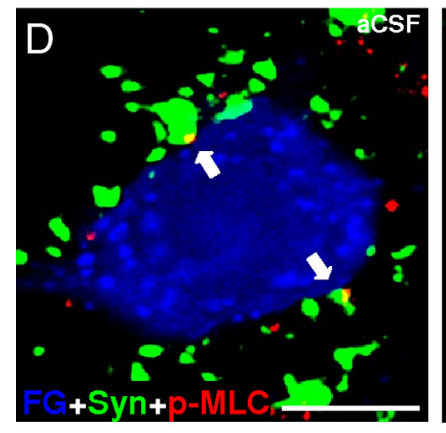
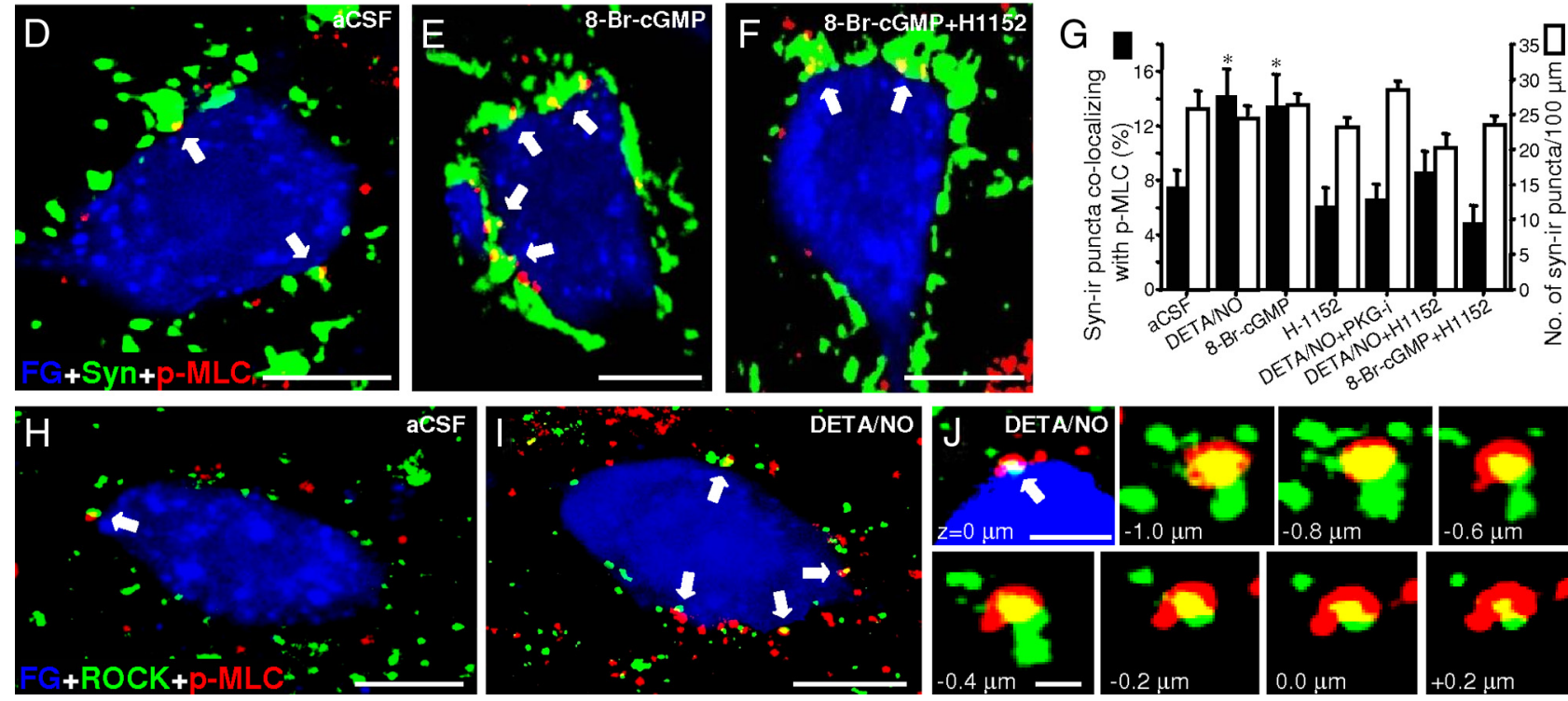

Figure 3. NO/cGMP/PKG induces p-MLC in synaptic puncta in a ROCK-dependent way before synapse loss. $\boldsymbol{A}$, Immunoblots for $\mathrm{p}$-MLC, MLC, and $\alpha$-tub performed from microdissected HN after incubation of slices for the indicated times with aCSF or DETA/NO-supplemented aCSF. $B$, Time course of the p-MLC/MLC ratio in HNs incubated with DETA/NO versus aCSF (aCSF and DETA/NO, $n=$ 3 Western blots from 6 animals per time point and condition). aCSF values was taken as 1 . ${ }^{*} p<0.05$, nonparametric Mann-Whitney $U$ test. ${ }^{\#} p<0.0001$, unpaired two-tailed Student's $t$ test. $\boldsymbol{C}$, Average of the indicated ratios after DETA/NO treatment for $4 \mathrm{~h}$ compared with the control condition taken as 1 . ${ }^{*} p<0.05$, nonparametric Mann-Whitney $U$ test. $\boldsymbol{D}-\boldsymbol{F}$, Syn- and $p-M L C$-ir puncta around FG-identified HMNs from slices incubated for $3 \mathrm{~h}$ under the indicated conditions. Secondary antibodies were labeled with Cy5 and Cy3 for immunolabeling of syn and p-MLC, respectively. $\mathbf{G}$, Average number of syn-ir puncta colocalizing with p-MLC-ir puncta (left $y$-axis) and syn-ir puncta per $100 \mu m$ of HMN perimeter (right $y$-axis) after receiving the indicated treatments (aCSF, $n=$ 20 HMNs; DETA/NO, $n=25$ HMNs; 8-Br-cGMP, $n=42$ HMNs; H1152, $n=24$ HMNs; DETA/NO + PKG-i, $n=23$ HMNs; DETA/NO +H1152, $n=20$ HMNs; 8-Br-cGMP+H1152, $n=21$ HMNs). Experiments were performed in four pups per condition. $\boldsymbol{H}-\boldsymbol{J}, \mathrm{ROCK}$ - and $\mathrm{p}$-MLC-ir puncta around FG-identified HMNs from slices incubated for $3 \mathrm{~h}$ under the indicated conditions. Details of the $z$-projection of a ROCK-ir puncta colocalizing with p-MLC $(\boldsymbol{J})$. Secondary antibodies were labeled with Cy 5 and Cy3 for immunolabeling of ROCK and p-MLC, respectively. ${ }^{*} p<0.05$, one-way ANOVA test relative to the aCSF condition. Error bars indicate SEM. Scale bars: $\boldsymbol{D}-\boldsymbol{J}, 10 \mu \mathrm{m}$; J, high-magnification photomicrographs, $1 \mu \mathrm{m}$.

trol, $3.7 \pm 0.1 \mathrm{~ms} ;$ DETA/NO, $4.6 \pm 0.5 \mathrm{~ms}$ ), these differences did not reach statistical significance. We recently reported that longterm incubation with DETA/NO had a direct effect on the intrinsic excitability of HMNs through inhibition of leak potassium currents, which leads to increases in their $R_{\mathrm{N}}$ (González-Forero et al., 2007). This purely postsynaptic action on $R_{\mathrm{N}}$ was also evidenced in the present study $(50.5 \pm 5.4 \mathrm{M} \Omega$ in control compared with $90.1 \pm 7.0 \mathrm{M} \Omega$ in DETA/NO-treated HMNs; $p<0.001$, unpaired two-tailed Student's $t$ test). Consequently, postsynaptic changes in membrane excitability induced by DETA/NO would be expected to augment the magnitude of voltage responses induced by synaptic currents. Therefore, our measurements of EPSP amplitude in current-clamp recordings most likely underestimated the action of NO on synaptic strength. Functional disturbance induced by chronic presence of the NO donor was also avoided by coaddition to the bath of ODQ or PKG-i (Fig. 2G,H). Thus, long-term action of NO/sGC/PKG pathway caused synaptic stripping accompanied by a reduction in synaptic strength in neonatal HMNs.

\section{NO-induced synapse elimination requires RhoA/ROCK} activity and involves previous MLC phosphorylation Since RhoA/ROCK is essential for neurite retraction in culture (Luo, 2000, 2002), we tested for the dependence of the NO- induced synaptic remodeling on RhoA/ROCK activity. Longterm inhibition of endogenous ROCK activity by adding either of two specific inhibitors, Y27632 or H1152, to the bath did not modify the linear density of syn-ir puncta on HMNs $(23.8 \pm 0.99$ and $27.3 \pm 1.19$ puncta/100 $\mu \mathrm{m}$, respectively) (Fig. $2 \mathrm{E}$ ). However, coaddition to the bath of ROCK inhibitors prevented reduction of the number of puncta induced by DETA/NO (Fig. $2 D, E$ ). These morphological findings were indeed consistent with the electrophysiological analysis of EPSPs. The amplitude of the evoked EPSP in HMNs after long-term coincubation of slices with DETA/NO plus either Y27632, H1152, or the Rho inhibitor Exo C3 was similar to that obtained in motoneurons from slices incubated in aCSF (Fig. 2G,H). These results strongly indicate that the synaptic reorganization induced in HMNs by activation of the NO/PKG cascade depends on RhoA/ROCK signaling.

ROCK can phosphorylate MLC, a proposed mechanism underlying actomyosin contraction and neurite outgrowth retraction (Ramakers, 2002; Newey et al., 2005). Immunoblot analysis revealed a time-dependent increase in the ratio of $\mathrm{p}$-MLC to MLC in DETA/NO- relative to aCSF-incubated $\mathrm{HN}$, reaching a maximal value 3-4 h after addition of the NO donor (Fig. $3 A, B$ ). Three hours after the beginning of treatment, DETA/NO induced a robust increase in the $\mathrm{p}-\mathrm{MLC}$ /MLC ratio; however, syn-ir puncta frequency remained unaltered (Fig. $3 A, B$ ). Therefore, in- 

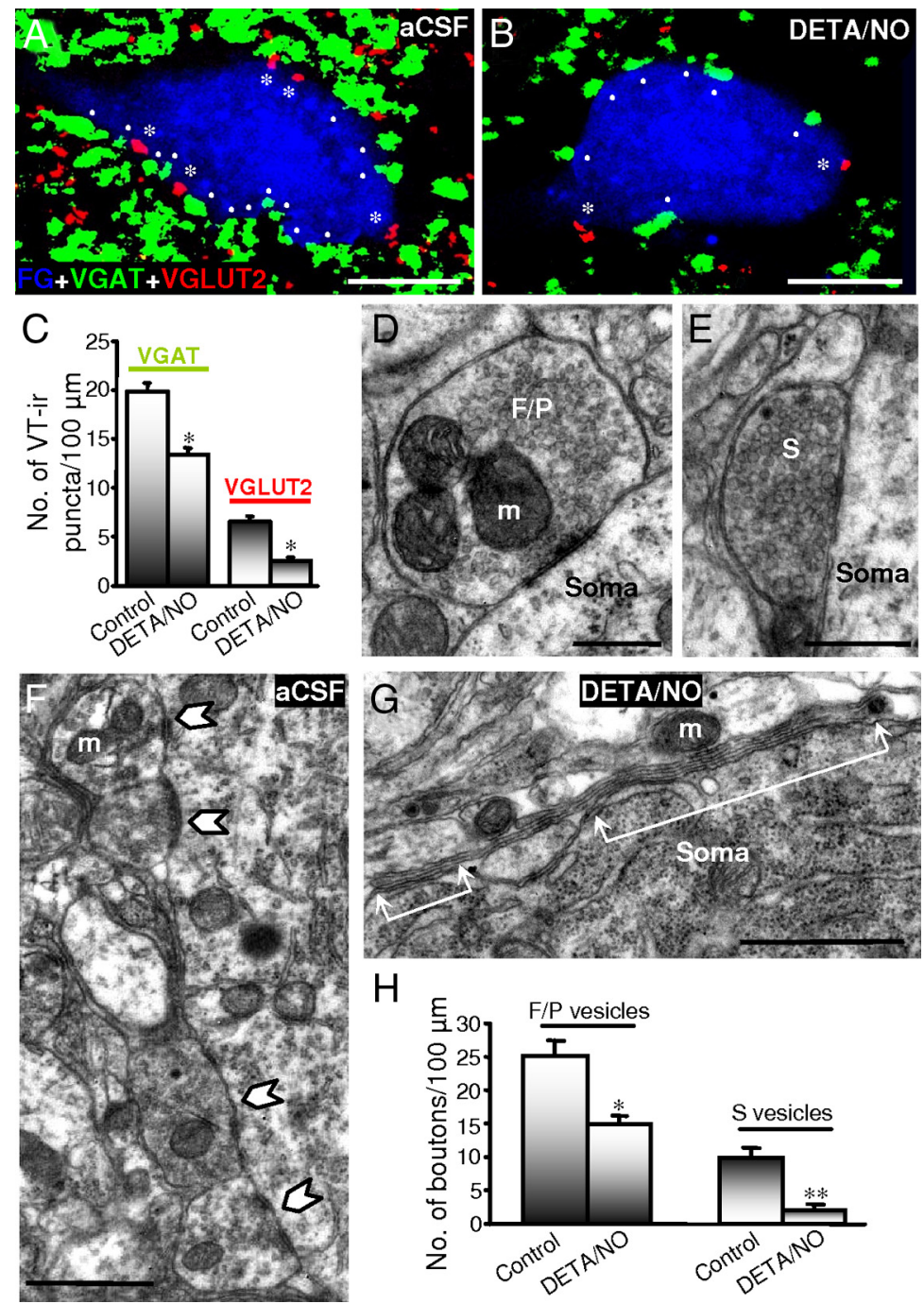

$\mathrm{H}$

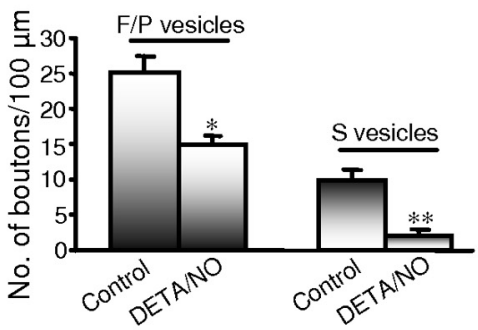

Figure 4. NO induced synapse detachment in neonatal motoneurons. $\boldsymbol{A}, \boldsymbol{B}, \mathrm{VGAT}$-ir $(\bullet)$ and VGLUT2-ir $\left(^{*}\right)$ puncta around FG-identified HMNs from slices incubated for $6 \mathrm{~h}$ under the indicated conditions. Secondary antibodies were labeled with Cy5 and Cy3 for immunolabeling of VGAT and VGLUT2, respectively. C, Average number of VT-ir puncta per $100 \mu \mathrm{m}$ of HMN perimeter after

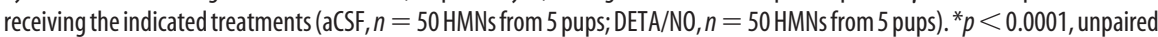
two-tailed Student's test. $\boldsymbol{D}, \boldsymbol{E}$, Illustrative examples of synaptic boutons attached to the plasma membrane of a HMN with $F / P(D)$ or $\boldsymbol{S}(\boldsymbol{E})$ vesicles. $\boldsymbol{F}, \boldsymbol{G}$, Segments of plasma membranes of HMN obtained from slices receiving the indicated treatments for $6 \mathrm{~h}$. The filled arrows point to synaptic boutons attached to the plasma membrane. In DETA/NO-treated HMNs, large segments of plasma membrane frequently were covered by glial-like processes (connected arrows). $\mathrm{m}$, Mitochondrion. $\boldsymbol{H}$, Average linear density of boutons, characterized by the type of vesicles, attached to motoneurons in slices receiving the indicated treatments (aCSF, $n=15$ HMNs from 3 pups; DETA/NO, $n=15$ HMNs from 3 pups). ${ }^{*} p<0.001$, ${ }^{* *} p<0.0001$, unpaired two-tailed Student's $t$ test. Error bars indicate SEM. Scale bars: $\boldsymbol{A}, \boldsymbol{B}, 10 \mu \mathrm{m} ; \boldsymbol{D}, \boldsymbol{E}, 0.5 \mu \mathrm{m} ; \boldsymbol{F}, \boldsymbol{G}, 1 \mu \mathrm{m}$.

crease in $\mathrm{p}$-MLC preceded syn-ir puncta reduction. In addition, at $6 \mathrm{~h}$ after DETA/NO treatment, the $\mathrm{p}-\mathrm{MLC} / \mathrm{MLC}$ ratio and the linear density of syn-ir puncta decreased in a similar proportion $(\sim 43 \%)$, suggesting that the two processes are closely related. The NO donor did not alter total amount of MLC in the HN after $4 \mathrm{~h}$ of incubation (Fig. 3C).

Subsequently, we analyzed syn-ir puncta coexpressing p-MLC after incubation for $3 \mathrm{~h}$ with different drugs (Fig. 3D-G). Incubation with DETA/NO or the cell-permeable cGMP analog 8-BrcGMP almost doubled the number of syn-ir puncta colocalizing with p-MLC-ir structures around HMNs (Fig. $3 D, G$ ). The NO/ cGMP-induced increase in $\mathrm{p}$-MLC was prevented by coaddition to the bath of PKG or ROCK inhibitors (Fig. $3 F, G$ ). Incubation with $\mathrm{H} 1152$ alone did not alter the amount of synaptic puncta colocalizing with p-MLC relative to the control condition (Fig.
3G). Thus, in our experimental conditions, p-MLC did not result from endogenous ROCK activity. Conclusively, DETA/NO incubation increased colocalization of ROCK-ir puncta with p-MLC-ir structures apposed to the HMN plasma membrane (control, $0.3 \pm 0.1$; DETA/NO, $1.1 \pm 0.3$ colocalizing puncta/100 $\mu \mathrm{m}$; $p=0.019$ ) (Fig. $3 H, I)$. Neither of these treatments significantly altered the frequency of syn-ir puncta apposed to HMNs (Fig. 3G). Together, our data suggest that NO promotes MLC phosphorylation through a ROCK-dependent mechanism by activating sGC and subsequently PKG before synapse loss.

\section{NO induces withdrawal of excitatory and inhibitory terminals from neonatal motoneurons}

Given that de novo nNOS expression affected excitatory, but not inhibitory, synapses in adult HMNs and the molecular mechanism was scrutinized using neonatal slices, we next looked for a NO-induced selective action at the neonatal stage for an age-dependent effect. This could be the basis for synaptic refinement occurring during postnatal development. Long-term incubation $(\sim 6 \mathrm{~h})$ of slices with DETA/NO led to a reduction in both VGLUT2- $(2.6 \pm$ 0.38 puncta/ $100 \mu \mathrm{m})$ and VGAT-ir (13.3 \pm 0.75 puncta $/ 100 \mu \mathrm{m}$ ) puncta apposed to the motoneuron membrane (Fig. 4A-C), even though excitatory were more profoundly affected than inhibitory synapses (VGLUT2, $-60.9 \pm 5.73 \%$; VGAT, $-32.8 \pm 3.77 \%$; $p<0.0001$, unpaired two-tailed Student's $t$ test). Electron microscopy analysis revealed that in HMNs from slices incubated with DETA/NO, the frequency of F/P- or S-type terminals was significantly reduced relative to the control aCSF condition (Fig. 4D-H). Although both subtypes of synaptic terminals were reduced, boutons containing $S$ vesicles $(-79.2 \pm 9.11 \%)$ were proportionally more affected than those with $\mathrm{F} / \mathrm{P}$ vesicles $(-40.6 \pm 5.04 \%)$. In DETA/ NO-treated motoneurons, we frequently observed a wide membrane that lacked attached terminals and was covered by glial-like processes (Fig. 4G). These results indicate that exogenous NO is able to induce synapse withdrawal within just $6 \mathrm{~h}$. NO affects both excitatory and inhibitory synapses, with a more prominent effect on excitatory boutons contacting neonatal motoneurons in vitro.

Age-dependent paracrine action of NO on motoneuron loss of excitatory and inhibitory puncta

NO shows differential selectivity for adult and neonatal motoneurons. One key question is whether this selectivity is acquired during postnatal development or results from differences in the NO sources provided at each stage. To evaluate these possibilities, we injected Av-eGFP/Av-nNOS into the tip of the 
tongue of neonatal rats (P3). Four to $6 \mathrm{~d}$ after adenoviral administration, VGLUT2and VGAT-ir puncta were significantly and similarly reduced compared with AveGFP-transfected animals (VGAT, $-66.3 \pm$ 3.31\%; VGLUT2, $-63.6 \pm 5.85 \%$ ) (Fig. $5 A-C)$. These data point to an agedependent change in the vulnerability of synapses to NO.

We next investigated whether NO-induced synaptic elimination involved a paracrine/retrograde action. Av-eGFP/ Av-nNOS-transfected rat pups were treated daily with the NOS inhibitor L-NAME, beginning the day of adenoviral administration to minimize NO synthesis until slice extraction. The linear density of syn-ir puncta apposed to HMNs, obtained at P7 and incubated for $6 \mathrm{~h}$ in aCSF, was reduced $(-47.1 \pm 5.8 \%)$ compared with FG-labeled HMNs incubated under the same conditions (Fig. 5D, G). This effect was similar to that evoked by long-term incubation with DETA/NO ( $-48.9 \pm 1.7 \%)$, indicating that similar and/or saturating levels of NO could be achieved in both experimental models. However, under the continuous presence of L-NAME or the membrane-impermeable NO scavenger C-PTIO (Ko and Kelly, 1999), the reduction in the frequency of syn-ir puncta was avoided (Fig. 5E-G).

Functional approaches confirmed these results. The amplitude of evoked EPSPs obtained from eGFP-positive HMNs (Fig. $5 \mathrm{H}$ ) was reduced $(-66.7 \pm 7.4 \%)$ relative to nontransfected HMNs when slices were maintained for $5-6 \mathrm{~h}$ in aCSF. However, incubation with L-NAME or C-PTIO prevented EPSP attenuation $(-3.7 \pm 15.0$ and $+13.9 \pm 20.2 \%$, respectively) (Fig. $5 I, J$ ). These data indicate that synaptic reorganization around cotransfected HMNs resulted from NO synthesis during the incubation period after slice extraction, requiring a paracrine/retrograde action of $\mathrm{NO}$ on target structures.

\section{NO-mediated synaptic withdrawal in a model of motoneuron pathology: motor nerve injury}

XIIth nerve crushing induced both de novo expression of nNOS and a NO/cGMP-dependent synaptic loss on adult HMNs (Sunico et al., 2005). One week after crushing, animals treated daily with the inactive stereoisomer D-NAME had a reduction in the frequency of VGLUT2- (3.4 \pm 0.29 puncta/100 $\mu \mathrm{m})$, but not in VGAT-ir (15.2 \pm 0.60 puncta/100 $\mu \mathrm{m})$ puncta compared with the intact side (VGLUT2, $6.5 \pm 0.45$ puncta/100 $\mu \mathrm{m}$; VGAT, $14.5 \pm 0.72$ puncta $/ 100 \mu \mathrm{m}$ ) (Fig. $6 A, B, D)$. In contrast, chronic administration of the NOS inhibitor L-NAME avoided reduction in excitatory puncta (VGLUT2, $6.7 \pm 0.38$ puncta/100 $\mu \mathrm{m}$; VGAT, $15.9 \pm 0.46$ puncta/ $100 \mu \mathrm{m}$ ) (Fig. $6 D$ ). Protective effects on the synaptic array were also obtained after nNOS or sGC inhibition with 7-NI or ODQ, respectively (Fig. 6C,D). We next looked for glutamatergic inputs containing ROCK as feasible targets for the paracrine NO action. One week after unilateral XIIth nerve injury, in the intact (control) HN, ROCK-ir puncta of both isoforms, ROCK $\alpha$ and ROCK $\beta$, were observed colocalizing and in close proximity to VGLUT2-ir puncta apposed to HMNs (Fig. $6 E)$. This agrees with the regulatory role of ROCK in cytoskeleton dynamics and microtubule organization (Benarroch, 2007). We found a dramatic decrease in the number of VGLUT2-ir related to ROCK-ir puncta on the injured side (VGLUT2/ROCK $\alpha$, $-79.0 \pm 8.9 \%$; VGLUT2/ROCK $\beta,-64.6 \pm 14.3 \%$ ) (Fig. $6 E, F)$. Furthermore, the number of $\mathrm{p}$-MLC-ir puncta in close proximity to injured HMNs increased at $4 \mathrm{~d}$ after injury (intact side, $3.5 \pm$ 0.6 ; injured side, $7.6 \pm 1.2$ puncta $100 \mu \mathrm{m} ; p<0.01$, unpaired two-tailed Student's $t$ test; $n=18 \mathrm{HMNs}$ from 3 rats per condition). At this same time point, the percentage of syn-ir colocalizing with p-MLC-ir puncta apposed to injured HMNs considerably increased (intact side, $11.4 \pm 2.5 \%$; injured side, $29.3 \pm 3.3 \%$; $p<0.0001$, unpaired two-tailed Student's $t$ test) (Fig. 6G,H). We selected this postinjury time window because nNOS was already upregulated (Sunico et al., 2005) and syn-ir reduction had not yet occurred (intact side, $16.9 \pm 0.55$; injured side, $17.2 \pm 0.7$ puncta/ $100 \mu \mathrm{m})$. These results suggest that, after motor nerve injury in adult rats, $\mathrm{NO}$, through sGC, mediates the loss of most ROCK-containing excitatory inputs, likely by a mechanism involving previous p-MLC.

\section{Discussion}

Our results indicate that nNOS upregulation in motoneurons is sufficient to induce synapse detachment at adult and neonatal 

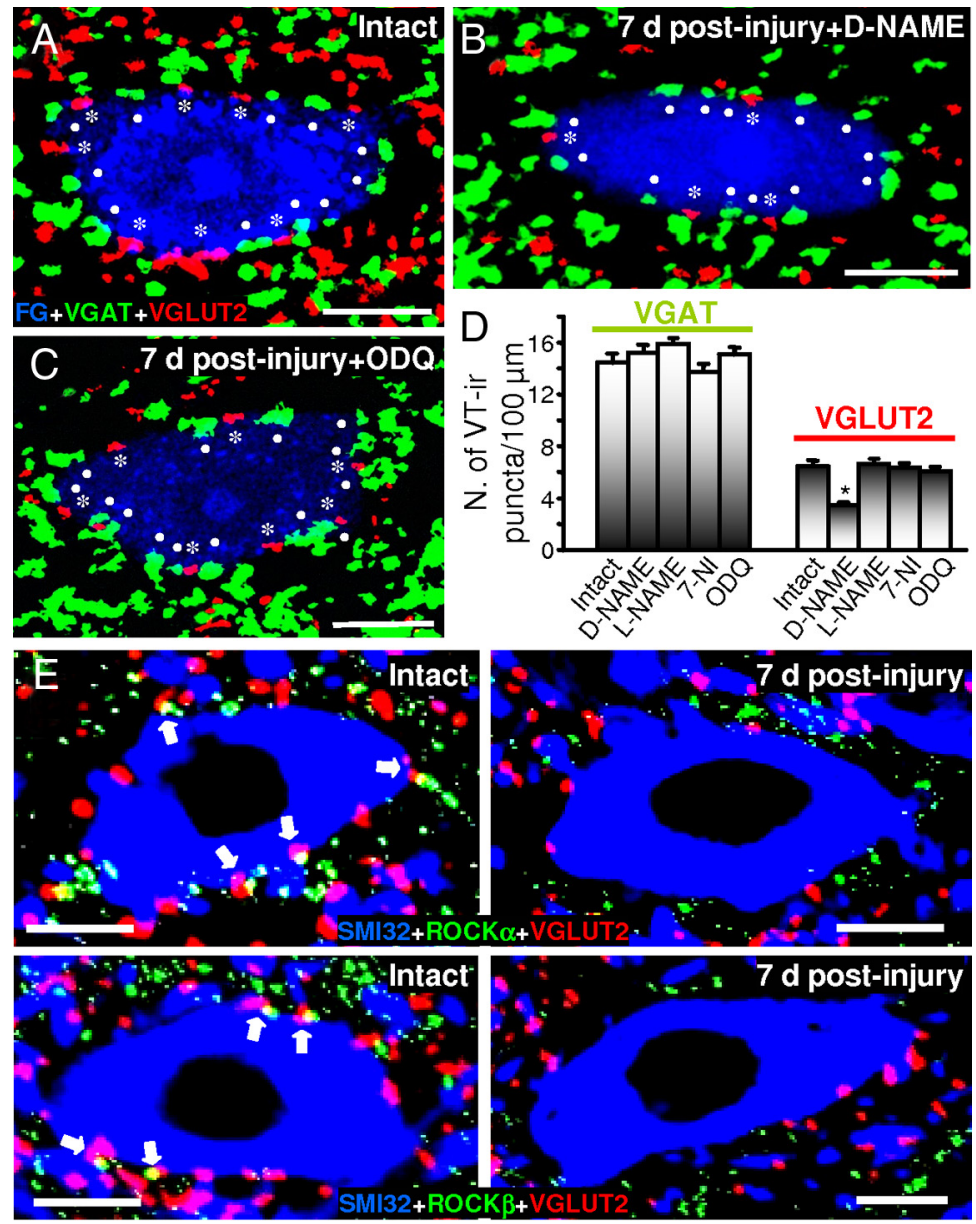

$\mathrm{F}$
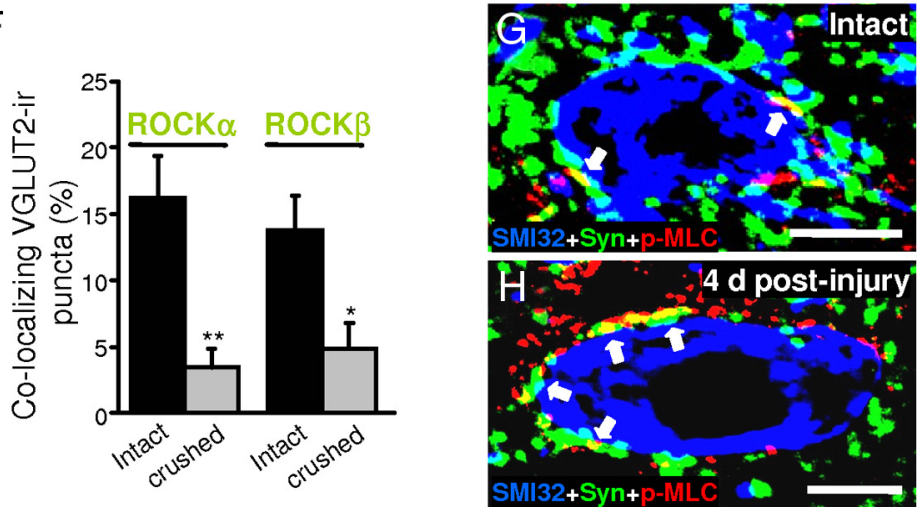

Figure 6. Axonal injury induces a NO/CGMP-dependent reduction of VGLUT2/ROCK-ir puncta apposed to adult motoneurons preceded by an increase in p-MLC. $\boldsymbol{A}$-C, VGAT-ir $(\bullet)$ and VGLUT2-ir $\left(^{*}\right)$ puncta around FG-backlabeled HMNs obtained from intact animals $(\boldsymbol{A})$ and $7 \mathrm{~d}$ after XIlth nerve crushing treated daily with the indicated drugs. Secondary antibodies were labeled with Cy5 and Cy3 for immunolabeling ofVGAT and VGLUT2, respectively. $\boldsymbol{D}$, Average number of VT-irpuncta per $100 \mu \mathrm{m}$ of HMN perimeter in FG-identified motoneurons $7 \mathrm{~d}$ after Xllth nerve crushing from animals receiving the indicated treatments (control/intact, $n=34 \mathrm{HMNs}$; D-NAME, $n=45 \mathrm{HMNs}$; L-NAME, $n=$ $33 \mathrm{HMNs} ; 7-\mathrm{Nl}, n=38 \mathrm{HMNs}$; $0 \mathrm{DQ}, n=30 \mathrm{HMNs}$; from 3 animals per experimental condition). ${ }^{*} p<0.0001$, one-way ANOVA test relative to the control/intact group. $E$, Syn-ir and ROCK $\alpha$ - or ROCK $\beta$-ir puncta adjacent to SMI32-immunolabeled HMNs in the intact and crushed sides $7 \mathrm{~d}$ after Xllth nerve crushing. Secondary antibodies were labeled with Cy5 and Cy3 for immunolabeling of ROCK and VGLUT2, respectively. $\boldsymbol{F}$, Average percentage of VGLUT2-ir puncta colocalizing with the indicated ROCK isoforms in the intact and crushed sides apposed to SMI32-identified HMNs $7 \mathrm{~d}$ after nerve injury ( $n=30$ HMNs per condition and ROCK isoform). ${ }^{*} p<0.01,{ }^{* *} p<0.001$ unpaired two-tailed Student's ttest. ErrorbarsindicateSEM. G, H, Syn-ir colocalizing with p-MLC puncta apposed to SMI32-immunolabeled HMNs in intact and injured sides from animals 4 d after unilateral Xllth nerve crushing. Secondary antibodies were labeled with Cy5 and Cy3 for immunolabeling of syn and p-MLC, respectively. Scale bars, $10 \mu \mathrm{m}$.

stages. Paracrine action of NO on presynaptic structures, through sGC/PKG and RhoA/ROCK signaling, triggered synapse loss preceded by MLC phosphorylation. We contribute evidence that this molecular cascade was operational in a model of motoneuron pathology: after traumatic injury of a motor nerve. Gain in NO sources and/or synthesis could be a general mechanism targeting synapses expressing sGC/PKGRhoA/ROCK, which are selectively removed during brain development and early stages of several neurodegenerative diseases.

Transduction of adult motoneurons with eGFP plus nNOS induced a selective reduction in the linear density of VGLUT2-ir puncta and S-type boutons. Paradoxically, the array of VGAT-ir puncta or F/P-type boutons apposed to HMNs was unaltered. Thus, nNOS transfection mimicked the effect of axonal injury on the synaptic coverage of HMNs (i.e., a selective decrease in VGLUT2-ir puncta density and larger loss of S-type boutons) (Sumner, 1975). However, it can be argued that nNOS upregulation in the muscle after adenoviral injection could actually trigger central synapse loss suffered by motoneurons. Notably, systemic nNOS inhibition prevented the effects of nerve damage on the synaptic arrangement even though neuromuscular communication was not yet reestablished at the tested time point (Sunico et al., 2008). In slices obtained from neonatal eGFP plus nNOS-transfected pups chronically receiving L-NAME, reduction of syn-ir puncta apposed to HMNs occurred after long-term incubation in aCSF, but not in L-NAME- or C-PTIO (a NO scavenger)supplemented medium. This indicates that NO from the transfected motoneuron induced synapse alterations through a paracrine/retrograde action. However, whether this paracrine action is sufficient remains elusive. Additionally, slices chronically incubated with DETA/NO, and therefore deprived of muscle influence, induced a strong decrease in VGATand VGLUT2-ir puncta apposed to and in $\mathrm{F} / \mathrm{P}$ - and S-types boutons attached to motoneurons. Together, these findings strongly indicate that gain in neuronal NO signaling is sufficient to induce synapse detachment in adult and neonatal motoneurons.

The molecular cascade involved in NO-induced synapse elimination was investigated in vitro. Synapse loss induced in neonatal slices by long-term incubation with DETA/NO was prevented by coincubation with a sGC inhibitor. The preventive effect of the sGC inhibitor was also observed after motor nerve injury in adults. Therefore, NO-induced synaptic detachment is mediated by a cGMP-dependent mechanism in in vitro and in vivo preparations as well as at neonatal and adult stages. The NONOatedirected effect was also prevented by coaddition of a specific PKG 
inhibitor to the bath, thereby involving the cGMP/PKG pathway in NO-induced synapse destabilization. The molecular machinery to support a sGC/PKG-mediated action of NO has been reported to be present in motoneurons and surrounding fibers (Furuyama et al., 1993; de Vente et al., 2001; Lin and Talman, 2005; GonzálezForero et al., 2007); this agrees with the increase in cGMP levels that occurs in motoneurons, fibers, and bouton-like structures in the $\mathrm{HN}$ by perfusing adult rats with a NO donor (Montero et al., 2008). These data agree with previous studies that implicated cGMP/PKG in mediating collapse responses in retinal and ganglionar growth cones to ephrin-B1 and Semaphorin $3 \mathrm{~A}$, respectively (Dontchev and Letourneau, 2002; Mann et al., 2003).

Synaptic withdrawal might involve actin cytoskeleton reorganization at the presynaptic component. Accordingly, NO/ cGMP/PKG action can be mediated by RhoA/ROCK signaling, directing changes in actin cytoskeleton and mediating neurite retraction by direct or indirect MLC phosphorylation (Luo, 2000, 2002; EtienneManneville and Hall, 2002). In this way, slice incubation with a Rho inhibitor or either of the two specific ROCK inhibitors fully avoided NO-induced decrease in the evoked EPSP in HMNs. ROCK inhibitors also prevented syn-ir puncta reduction by $\mathrm{NO}$, although incubation with either of these inhibitors alone did not modify syn-ir puncta density around motoneurons. Furthermore, incubation for $3 \mathrm{~h}$ with the NONOate increased MLC phosphorylation in the neonatal HN and the syn-ir puncta colocalizing with p-MLC apposed to motoneurons, although syn-ir coverage remained unaffected. The latter was mimicked by adding a cGMP analog to the bath and was prevented by coincubation with PKG or ROCK inhibitors. However, ROCK inhibitors per se did not modulate this parameter, thus minimizing the possibility of a contaminating effect of basal ROCK activity on MLC phosphorylation. This observation is supported by the increase in ROCK and p-MLC colocation under DETA/NO treatment. Therefore, NO/cGMP/PKG-induced synapse remodeling is dependent on RhoA/ROCK signaling, involving phosphorylation of the ROCK substrate MLC before synapse withdrawal. Increased RhoA/ROCK activity resulting from long-term action of the NO/cGMP/PKG cascade has been previously suggested in arterial smooth muscle cells (Sauzeau et al., 2003), in the penis (Bivalacqua et al., 2007), and in hearts from diabetic rats (Soliman et al., 2008). In this sense, cGMP/PKG together with ROCK and/or MLC-kinase mediates growth cone collapse responses to ephrin-B1, -A5, and Semaphorin 3A in cells from different origins (Dontchev and Letourneau, 2002; Mann et al., 2003; Yue et al., 2008). The function of MLC-kinase is likely to be mediated through MLC phosphorylation, leading to myosin activation and actomyosin contraction (Luo, 2002).

Why does NO induce loss of excitatory and inhibitory synapses at neonatal stages, but only affect excitatory ones in adult- hood? We know that NO participates in developmental synaptic refinement (Wu et al., 1994) and, at neonatal stages, motoneurons transiently express nNOS that fully disappear before P21 (Vazquez et al., 1999; Gao et al., 2008). Synaptic refinement of afferent inputs on HMNs during maturation could involve specific loss of NO-sensitive GABAergic inputs. Interestingly, VGAT-, but not VGLUT2-ir, puncta are reduced in adulthood relative to neonatal stage. This decrease, occurring during postnatal maturation, is similar to that induced by DETA/NO incubation in neonatal motoneurons. Accordingly, a high proportion of GABAergic synapses disappear from P8 to P14 in the nucleus of tractus solitarii (Torrealba and Müller, 1999; Yoshioka et al., 2006). A differential sensitivity in the potentiation of glutamatergic EPSPs and GABAergic IPSPs to the NO/cGMP pathway has been described in this nucleus, reaching levels of saturation near 15 nM NO (Wang et al., 2007). This is higher than maximal endogenous nNOS-derived $\sim 4 \mathrm{nM}$ NO from cerebellar neurons in vitro (Bellamy et al., 2002) or basal $<10 \mathrm{~nm}$ NO in the rat cerebral tissue, which increases up to $4 \mu \mathrm{M}$ (pathological) after ischemia (Malinski et al., 1993). Then, under physiological nanomolar concentrations of $\mathrm{NO}$, probably reached during developmental maturation, a preferential loss of $\mathrm{NO}$-sensitive inhibitory synapse could occur. However, pathological micromolar concentrations 
of NO, as achieved under incubation with $1 \mathrm{~mm}$ DETA/NO $(\sim 1$ $\mu \mathrm{M} \mathrm{NO}$ ) (Abudara et al., 2002), by hCMV-directed nNOS overexpression in motoneurons or in pathological nNOS upregulation induced by motoneuron axonal injury, could induce additional effects on NO-sensitive excitatory synapses. Nonetheless, we cannot dismiss the possibility of changes in presynaptic molecular composition during postnatal maturation lowering the NO threshold of excitatory inputs.

Axotomized HMNs upregulate nNOS and undergo excitatory synaptic stripping, which likely accounts for the strong reduction in their firing activities (Sunico et al., 2005). Systemic administration of inhibitors of the neuronal NO/cGMP pathway prevented synapse loss. Motor nerve injury induced an increase in the number of syn/p-MLC-positive puncta, before synapse detachment, followed by a strong reduction in VGLUT2/ROCKpositive puncta around adult injured HMNs. A decrease in synaptic density together with overexpression and/or de novo expression of nNOS are early common hallmarks in several neurodegenerative diseases. In the spinal cord of SOD ${ }^{\mathrm{G} 93 \mathrm{~A}}$ mice, ROCK $\alpha$ is overexpressed (Hu et al., 2003), suggesting an increase in RhoA/ROCK signaling. In addition, a ROCK inhibitor has been recently described as a therapeutic lead in HD (Shao et al., 2008). Interestingly, the pattern of neurodegeneration in the AD brain is consistent with a model in which synaptic scaling is the driving force for disease progression (Small, 2008). A selective elimination of glutamatergic synapses on striatopallidal neurons occurs in PD models (Day et al., 2006), a promising connection with what happens in adult nNOS-expressing motoneurons. These additional data support the possibility that the action mechanism underlying synapse loss described here could be common to several neurodegenerative diseases.

We propose here a mechanism of action triggered by nNOS upregulation in the course of diverse pathological conditions of the nervous system underlying synapse reduction (Fig. 7). NO production is coupled with $\mathrm{Ca}^{2+}$ influx through NMDA subtype receptor (Garthwaite et al., 1988) and is also effected by nonNMDA receptor agonists (Wood et al., 1990). Axonal injury of motoneurons increases NMDA expression and completely depletes the GluR2 subunit of AMPA receptors (García Del Caño et al., 2000), which prevents $\mathrm{Ca}^{2+}$ influx through them (Hollmann et al., 1991). Thus, in impaired neurons, more sources are available to increase intracellular $\mathrm{Ca}^{2+}$ concentration, which in turn may facilitate NO production via $\mathrm{Ca}^{2+} /$ calmodulin-mediated nNOS activation (Fig. 7). As a gas, pathological NO from sick neurons can, by a paracrine action, induce retraction of neighboring synapses whether they have the appropriate molecular substrate. This could explain why not all synapses are lost in pathological conditions around nNOS-expressing neurons. NO activating sGC increases intrasynaptic cGMP level that, in turn, activates PKG. Directly or indirectly, PKG stimulates RhoA/ ROCK signaling. ROCK activation results in MLC phosphorylation either by a direct action on MLC and/or by activation of MLC-kinase and/or by inhibition of MLC-phosphatase (Benarroch, 2007). Subsequently, $\mathrm{p}$-MLC, as a regulatory factor, favors actomyosin contraction, which eventually contributes to bouton retraction.

\section{References}

Abudara V, Alvarez AF, Chase MH, Morales FR (2002) Nitric oxide as an anterograde neurotransmitter in the trigeminal motor pool. J Neurophysiol 88:497-506.

Anneser JM, Cookson MR, Ince PG, Shaw PJ, Borasio GD (2001) Glial cells of the spinal cord and subcortical white matter up-regulate neuronal nitric oxide synthase in sporadic amyotrophic lateral sclerosis. Exp Neurol 171:418-421.
Bellamy TC, Griffiths C, Garthwaite J (2002) Differential sensitivity of guanylyl cyclase and mitochondrial respiration to nitric oxide measured using clamped concentrations. J Biol Chem 277:31801-31807.

Benarroch EE (2007) Rho GTPases: role in dendrite and axonal growth, mental retardation, and axonal regeneration. Neurology 68:1315-1318.

Bivalacqua TJ, Liu T, Musicki B, Champion HC, Burnett AL (2007) Endothelial nitric oxide synthase keeps erection regulatory function balance in the penis. Eur Urol 51:1732-1740.

Bodian D (1966) Electron microscopy: two major synaptic types on spinal motoneurons. Science 151:1093-1094.

Catania MV, Aronica E, Yankaya B, Troost D (2001) Increased expression of neuronal nitric oxide synthase spliced variants in reactive astrocytes of amyotrophic lateral sclerosis human spinal cord. J Neurosci 21:RC148(1-5).

Centonze D, Muzio L, Rossi S, Cavasinni F, De Chiara V, Bergami A, Musella A, D’Amelio M, Cavallucci V, Martorana A, Bergamaschi A, Cencioni MT, Diamantini A, Butti E, Comi G, Bernardi G, Cecconi F, Battistini L, Furlan R, Martino G (2009) Inflammation triggers synaptic alteration and degeneration in experimental autoimmune encephalomyelitis. J Neurosci 29:3442-3452.

Cepeda C, Wu N, André VM, Cummings DM, Levine MS (2007) The corticostriatal pathway in Huntington's disease. Prog Neurobiol 81:253-271.

Day M, Wang Z, Ding J, An X, Ingham CA, Shering AF, Wokosin D, Ilijic E, Sun Z, Sampson AR, Mugnaini E, Deutch AY, Sesack SR, Arbuthnott GW, Surmeier DJ (2006) Selective elimination of glutamatergic synapses on striatopallidal neurons in Parkinson disease models. Nat Neurosci 9:251-259.

Deckel AW, Tang V, Nuttal D, Gary K, Elder R (2002) Altered neuronal nitric oxide synthase expression contributes to disease progression in Huntington's disease transgenic mice. Brain Res 939:76-86.

de Vente J, Asan E, Gambaryan S, Markerink-van Ittersum M, Axer H, Gallatz K, Lohmann SM, Palkovits M (2001) Localization of cGMP-dependent protein kinase type II in rat brain. Neuroscience 108:27-49.

Dontchev VD, Letourneau PC (2002) Nerve growth factor and semaphorin $3 \mathrm{~A}$ signaling pathways interact in regulating sensory neuronal growth cone motility. J Neurosci 22:6659-6669.

Emre M (2003) Dementia associated with Parkinson's disease. Lancet Neurol 2:229-237.

Etienne-Manneville S, Hall A (2002) Rho GTPases in cell biology. Nature 420:629-635.

Eve DJ, Nisbet AP, Kingsbury AE, Hewson EL, Daniel SE, Lees AJ, Marsden CD, Foster OJ (1998) Basal ganglia neuronal nitric oxide synthase mRNA expression in Parkinson's disease. Brain Res Mol Brain Res 63:62-71.

Fernández-Vizarra P, Fernández AP, Castro-Blanco S, Encinas JM, Serrano J, Bentura ML, Muñoz P, Martínez-Murillo R, Rodrigo J (2004) Expression of nitric oxide system in clinically evaluated cases of Alzheimer's disease. Neurobiol Dis 15:287-305.

Furuyama T, Inagaki S, Takagi H (1993) Localizations of alpha 1 and beta 1 subunits of soluble guanylate cyclase in the rat brain. Brain Res Mol Brain Res 20:335-344.

Gao S, Cheng C, Zhao J, Chen M, Li X, Shi S, Niu S, Qin J, Lu M, Shen A (2008) Developmental regulation of PSD-95 and nNOS expression in lumbar spinal cord of rats. Neurochem Int 52:495-501.

García Del Caño G, Gerrikagoitia I, Sarasa M, Matute C, Martínez-Millán L (2000) Ionotropic glutamate receptor subunits are differentially regulated in the motoneuronal pools of the rat hypoglossal nucleus in response to axotomy. J Neurocytol 29:509-523.

Garthwaite J, Charles SL, Chess-Williams R (1988) Endothelium-derived relaxing factor release on activation of NMDA receptors suggests role as intercellular messenger in the brain. Nature 336:385-388.

González-Forero D, Portillo F, Sunico CR, Moreno-López B (2004) Nerve injury reduces responses of hypoglossal motoneurones to baseline and chemoreceptor-modulated inspiratory drive in the adult rat. J Physiol 557:991-1011.

González-Forero D, Portillo F, Gómez L, Montero F, Kasparov S, MorenoLópez B (2007) Inhibition of resting potassium conductances by longterm activation of the $\mathrm{NO} / \mathrm{cGMP} /$ protein kinase G pathway: a new mechanism regulating neuronal excitability. J Neurosci 27:6302-6312.

Gray EG (1959) Axo-somatic and axo-dendritic synapses of the cerebral cortex: an electron microscope study. J Anat 93:420-433.

Heredia L, Helguera P, de Olmos S, Kedikian G, Solá Vigo F, LaFerla F, Staufenbiel M, de Olmos J, Busciglio J, Cáceres A, Lorenzo A (2006) Phosphorylation of actin-depolymerizing factor/cofilin by LIM-kinase 
mediates amyloid $\beta$-induced degeneration: a potential mechanism of neuronal dystrophy in Alzheimer's disease. J Neurosci 26:6533-6542.

Hollmann M, Hartley M, Heinemann S (1991) $\mathrm{Ca}^{2+}$ permeability of KAAMPA-gated glutamate receptor channels depends on subunit composition. Science 252:851-853.

Hu JH, Chernoff K, Pelech S, Krieger C (2003) Protein kinase and protein phosphatase expression in the central nervous system of G93A mSOD over-expressing mice. J Neurochem 85:422-431.

Ikemoto A, Kawanami T, Llena JF, Hirano A (1994) Immunocytochemical studies on synaptophysin in the anterior horn of lower motor neuron disease. J Neuropathol Exp Neurol 53:196-201.

Ince PG, Slade J, Chinnery RM, McKenzie J, Royston C, Roberts GW, Shaw PJ (1995) Quantitative study of synaptophysin immunoreactivity of cerebral cortex and spinal cord in motor neuron disease. J Neuropathol Exp Neurol 54:673-679.

Kim HJ, Martemyanov KA, Thayer SA (2008) Human immunodeficiency virus protein Tat induces synapse loss via a reversible process that is distinct from cell death. J Neurosci 28:12604-12613.

Ko GY, Kelly PT (1999) Nitric oxide acts as a postsynaptic signaling molecule in calcium/calmodulin-induced synaptic potentiation in hippocampal CA1 pyramidal neurons. J Neurosci 19:6784-6794.

Lee J, Ryu H, Ferrante RJ, Morris SM Jr, Ratan RR (2003) Translational control of inducible nitric oxide synthase expression by arginine can explain the arginine paradox. Proc Natl Acad Sci U S A 100:4843-4848.

Lin LH, Talman WT (2005) Soluble guanylate cyclase and neuronal nitric oxide synthase colocalize in rat nucleus tractus solitarii. J Chem Neuroanat 29:127-136.

Luo L (2000) Rho GTPases in neuronal morphogenesis. Nat Rev Neurosci 1:173-180.

Luo L (2002) Actin cytoskeleton regulation in neuronal morphogenesis and structural plasticity. Annu Rev Cell Dev Biol 18:601-635.

Lüth HJ, Holzer M, Gertz HJ, Arendt T (2000) Aberrant expression of nNOS in pyramidal neurons in Alzheimer's disease is highly co-localized with p21ras and p16INK4a. Brain Res 852:45-55.

Malinski T, Bailey F, Zhang ZG, Chopp M (1993) Nitric oxide measured by a porphyrinic microsensor in rat brain after transient middle cerebral artery occlusion. J Cereb Blood Flow Metab 13:355-358.

Mann F, Miranda E, Weinl C, Harmer E, Holt CE (2003) B-type Eph receptors and ephrins induce growth cone collapse through distinct intracellular pathways. J Neurobiol 57:323-336.

Montero F, Portillo F, González-Forero D, Moreno-López B (2008) The nitric oxide/cyclic guanosine monophosphate pathway modulates the inspiratory-related activity of hypoglossal motoneurons in the adult rat. Eur J Neurosci 28:107-116.

Moreno-López B, González-Forero D (2006) Nitric oxide and synaptic dynamics in the adult brain: physiopathological aspects. Rev Neurosci 17:309-357.

Moreno-López B, Romero-Grimaldi C, Noval JA, Murillo-Carretero M, Matarredona ER, Estrada C (2004) Nitric oxide is a physiological inhibitor of neurogenesis in the adult mouse subventricular zone and olfactory bulb. J Neurosci 24:85-95.

Newey SE, Velamoor V, Govek EE, Van Aelst L (2005) Rho GTPases, dendritic structure, and mental retardation. J Neurobiol 64:58-74.

Palop JJ, Chin J, Mucke L (2006) A network dysfunction perspective on neurodegenerative diseases. Nature 443:768-773.

Pérez-Severiano F, Escalante B, Vergara P, Ríos C, Segovia J (2002) Agedependent changes in nitric oxide synthase activity and protein expression in striata of mice transgenic for the Huntington's disease mutation. Brain Res 951:36-42.

Petratos S, Li QX, George AJ, Hou X, Kerr ML, Unabia SE, Hatzinisiriou I,
Maksel D, Aguilar MI, Small DH (2008) The beta-amyloid protein of Alzheimer's disease increases neuronal CRMP-2 phosphorylation by a Rho-GTP mechanism. Brain 131:90-108.

Ramakers GJ (2002) Rho proteins, mental retardation and the cellular basis of cognition. Trends Neurosci 25:191-199.

Sasaki S, Maruyama S (1994) Synapse loss in anterior horn neurons in amyotrophic lateral sclerosis. Acta Neuropathol 88:222-227.

Sasaki S, Shibata N, Iwata M (2001) Neuronal nitric oxide synthase immunoreactivity in the spinal cord in amyotrophic lateral sclerosis. Acta Neuropathol 101:351-357.

Sauzeau V, Rolli-Derkinderen M, Marionneau C, Loirand G, Pacaud P (2003) RhoA expression is controlled by nitric oxide through cGMPdependent protein kinase activation. J Biol Chem 278:9472-9480.

Shao J, Welch WJ, Diprospero NA, Diamond MI (2008) Phosphorylation of profilin by ROCK1 regulates polyglutamine aggregation. Mol Cell Biol 28:5196-5208.

Simic G, Lucassen PJ, Krsnik Z, Kruslin B, Kostovic I, Winblad B, Bogdanovic $\mathrm{N}$ (2000) nNOS expression in reactive astrocytes correlates with increased cell death related DNA damage in the hippocampus and entorhinal cortex in Alzheimer's disease. Exp Neurol 165:12-26.

Small DH (2008) Network dysfunction in Alzheimer's disease: does synaptic scaling drive disease progression? Trends Mol Med 14:103-108.

Soliman H, Craig GP, Nagareddy P, Yuen VG, Lin G, Kumar U, McNeill JH, Macleod KM (2008) Role of inducible nitric oxide synthase in induction of RhoA expression in hearts from diabetic rats. Cardiovasc Res 79: 322-330.

Sumner BE (1975) A quantitative analysis of boutons with different types of synapse in normal and injured hypoglossal nuclei. Exp Neurol 49:406-417.

Sunico CR, Portillo F, González-Forero D, Moreno-López B (2005) Nitric oxide-directed synaptic remodeling in the adult mammal CNS. J Neurosci 25:1448-1458.

Sunico CR, Portillo F, González-Forero D, Kasparov S, Moreno-López B (2008) Evidence for a detrimental role of nitric oxide synthesized by endothelial nitric oxide synthase after peripheral nerve injury. Neuroscience 157:40-51.

Torrealba F, Müller C (1999) Ultrastructure of glutamate and GABA immunoreactive axon terminals of the rat nucleus tractus solitarius, with a note on infralimbic cortex afferents. Brain Res 820:20-30.

Uchizono K (1965) Characteristics of excitatory and inhibitory synapses in the central nervous system of the cat. Nature 207:642-643.

Vazquez C, Anesetti G, Martinez Palma L (1999) Transient expression of nitric oxide synthase in the hypoglossal nucleus of the rat during early postnatal development. Neurosci Lett 275:5-8.

Wang S, Paton JF, Kasparov S (2007) Differential sensitivity of excitatory and inhibitory synaptic transmission to modulation by nitric oxide in rat nucleus tractus solitarii. Exp Physiol 92:371-382.

Wood PL, Emmett MR, Rao TS, Cler J, Mick S, Iyengar S (1990) Inhibition of nitric oxide synthase blocks $N$-methyl-D-aspartate-, quisqualate-, kainate-, harmaline-, and pentylenetetrazole-dependent increases in cerebellar cyclic GMP in vivo. J Neurochem 55:346-348.

Wu HH, Williams CV, McLoon SC (1994) Involvement of nitric oxide in the elimination of a transient retinotectal projection in development. Science 265:1593-1596.

Yoshioka M, Tashiro Y, Inoue K, Kawai Y (2006) Postnatal development of GABAergic axon terminals in the rat nucleus of tractus solitarius. Brain Res 1107:111-120.

Yue X, Dreyfus C, Kong TA, Zhou R (2008) A subset of signal transduction pathways is required for hippocampal growth cone collapse induced by ephrin-A5. Dev Neurobiol 68:1269-1286. 